\title{
Service Quality in Tourism: A Systematic Literature Review and Keyword Network Analysis
}

\author{
Jinsoo Park ${ }^{1}\left(\mathbb{D}\right.$ and EuiBeom Jeong ${ }^{2, *} \mathbb{C}$ \\ 1 Department of Business Administration, The Catholic University of Korea, Jibong-ro, Bucheon-si, \\ Gyeonggi-do 14662, Korea \\ 2 Korea University Business School, Anam-ro, Seongbuk-gu, Seoul 02841, Korea \\ * Correspondence: euibeom@korea.ac.kr; Tel.: +82-2-3290-2805
}

Received: 29 May 2019; Accepted: 28 June 2019; Published: 3 July 2019

\begin{abstract}
The tourism industry has received increasing attention as it has become one of the fastest developing business sectors around the world. Service quality in tourism has come to be regarded as an important impetus for economic growth; however, the focus on tourism service quality has not yet been satisfactorily or comprehensively reviewed. Therefore, we conducted a systematic literature review combining bibliometric, citation network and keyword network analysis. We selected the top five tourism journals from the SCOPUS database and then collected papers according to their keywords. Our results revealed the critical issues, topics, and changes over time regarding research on tourism service quality. The critical issues included the important impact of service quality on tourist behavior and service quality evaluation, and topics comprised (1) tourist satisfaction, (2) sustainable issues in tourism, (3) value of service quality for customers, (4) restaurant service quality, (5) customers' perceptions of tourism, (6) service quality evaluation in tourism, and (7) service quality's influence on customer behavior. Furthermore, the keyword network analysis results revealed the most influential keywords.
\end{abstract}

Keywords: service quality; systematic literature review; keyword network analysis

\section{Introduction}

During the last few decades, the tourism industry has become extremely economically relevant, as it has become one of the fastest developing areas in the contemporary business environment. For example, global international tourist arrivals in 2013 reached a record of 1.087 billion, whereas international tourism revenue constituted a record US $\$ 1.159$ billion in the same year [1]. This shows that tourism can significantly contribute to the economic growth of tourist destinations by increasing employment opportunities, developing infrastructure, and attracting foreign exchange earnings [2]. Tourism's economic impetus can be investigated using various perspectives, which include, for example, (1) its direct effects such as "sales, employment, tax revenues, and income levels", which come from the immediate impacts of tourist spending [2-4], (2) its indirect effects such as "prices, quantity and quality of products and services, taxes and property, and social and environmental impacts" [2-4], and (3) its efficiency and productivity due to economic resources being allocated to promote cost reductions in tourism sectors [2,5-7]. As a result, previous literature has regarded tourism as an important cause of variation in economic growth in many countries.

Among the factors related to tourism, service quality in tourism has received increasing academic attention. For example, research papers have been published in academic journals that utilize SERVQUAL, the most representative model for measuring service quality $[8,9]$. Nevertheless, service quality in tourism has not yet been satisfactorily reviewed despite its impact on tourists' destination choice. Furthermore, because of tourism's wide-ranging scope, which extends to various business 
settings, few studies have used a comprehensive perspective to examine service quality in tourism. Therefore, our study's main objective was to identify the most influential studies as well as both broad and specific issues regarding tourism service quality and explore this research field's current and future directions and trends through a systematic literature review.

\section{Literature Review}

Identifying the themes of a given field for the purpose of improving our understanding of it, and therefore stimulating further research is a proactive effort. Through the process of mapping and evaluating the body of literature, scholars can identify potential research gaps and opportunities for future research [10].

With regards to conducting such literature reviews, researchers have adopted various approaches. One influential perspective is the "popularity-based approach," which includes the technique of bibliometric analysis. This method was created to investigate authors' keywords and titles of published articles in various research fields. Specifically, bibliometric analysis can provide insights not captured or evaluated by other reviews because it offers additional data regarding authors, affiliation, popular words, and keywords and their frequency of use. However, while the popularity-based approach can indicate the importance of titles or keywords in a given research area by investigating their frequency of use in papers, significant information can only be obtained post-publication [11]. Moreover, this approach is considered unsuitable for identifying shared topical content due to its inability to assess relationships among published papers within a certain field.

Another literature review method involves the "network-based approach," which uses citation and co-citation analysis to investigate the network structure that exists among articles in a given field by mapping and visualizing the citations generated among papers. Citation analysis has been used to determine the popularity of a publication [12]; that is, a network analysis of commonly used citations aims to identify the popularity of a published paper by counting how frequently a paper is cited by other papers. Unlike citation analysis, co-citation analysis has been implemented to identify topics in a given area by constructing a co-citation network comprising a set of nodes (journal papers) and a set of links (co-occurrences of the papers in other papers). That is, if two publications appear together in other publications' reference lists, they represent a co-citation relationship [13]. Co-citation can be used to explore data structure by applying mapping as a form of exploratory data analysis. In other words, a co-citation network constructed based on papers that are more frequently co-cited indicates that the included papers have similar subject areas [14]. However, those network-based analyses have mainly been conducted on published papers, rather than specified keywords. Therefore, a network of commonly used citations or co-citations does not directly represent a specified knowledge network for a given area from a comprehensive perspective. To comprehensively identify issues and topics pertaining to tourism service quality, it is therefore essential to include papers not involved in the network.

Lai et al. [15] performed a systematic literature review on service quality in Hospitality and Tourism using the pathfinder network approach. This study differs from the present study in two significant ways. First, Lai et al. used citation counts to identify the most influential papers for content analysis. While citation counts are an important indicator of a paper's impact, a highly cited paper can not necessarily indicate a prestigious paper, as measured by the number of times a paper is cited by other highly cited papers. In contrast, we used PageRank in the present study as an indicator of both "popularity, measured by citation count, and "prestige" to identify the most impactful papers and to analyze the contents of selected papers. Second, Lai et al. conducted a network-based study involving citation and co-citation analysis to identify research gaps and suggest directions for future research. Here, we adopted a systematic literature review approach that combined a network-based approach with bibliometric and keyword network analysis. The results are significantly different.

Considering these perceived drawbacks in the existing literature, we resolved to conduct a systematic literature review that combined the traditional systematic literature review approach 
(bibliometric and citation analysis) with keyword network analysis for a more comprehensive evaluation of research on service quality in tourism. As part of our method, we identified and investigated the keyword network linking an author's keywords to comprehensively map knowledge of tourism service quality and identify important issues and topics and their change over time.

In our study, we first conducted rigorous bibliometric (i.e., frequency analysis) and network analysis on service quality in tourism research (e.g., citation, co-citation, and keyword network analysis) to map the knowledge structure of the issue and topic, and then carried out a content analysis of the key papers. In order to examine the structural characteristics of the network and identify critical issues and topics regarding service quality in tourism from a comprehensive perspective, we selected influential papers and extracted keywords. We performed a citation analysis to identify critical content in the existing literature and conducted a co-citation analysis to explore topical content based on classification of the existing studies. Finally, in order to carry out a systematic content analysis of the theme, we constructed a network based on keywords extracted from the selected publications and investigated changes in important keywords over time.

\section{Research Methodology}

As a first step, we selected tourism-related journals from the SCOPUS database. To extract the most influential papers, we reduced the journals to only the top five using the categories and impact factor (SJR, SCImago journal rank) provided by SCOPUS. We used "service quality" as the main search keyword.

We located papers with the identified keywords from the selected major journals by using the "article title, abstract, keywords" search in SCOPUS. To select the most influential papers, only journal articles written in English were used; conference papers, book series, commercial publications, and magazine articles were excluded. Search results included essential information such as title, author(s), abstract, paper keywords, and references.

Given that various tourism scholars might have differing perspectives in terms of which journals publish tourism-related articles, this study attempted to collect data from a diverse range of top tourism journals. The initial search attempts resulted in 178 papers. Due to a higher number of published papers in 2007 compared to 2008, we collected papers published during a 12-year period from 2008 to 2019. The results from the selected journals are summarized in Table 1. Figure 1 shows the quantity of publications per year.

Table 1. Selected journals (2008-2019).

\begin{tabular}{|c|c|c|c|c|c|c|c|c|c|c|c|c|c|}
\hline Journals & 2008 & 2009 & 2010 & 2011 & 2012 & 2013 & 2014 & 2015 & 2016 & 2017 & 2018 & 2019 & Total \\
\hline Annals of Tourism Research & & & & & 1 & 1 & & & & & & & 2 \\
\hline $\begin{array}{c}\text { International Journal } \\
\text { of Hospitality Management }\end{array}$ & 4 & 4 & 8 & 9 & 13 & 11 & 9 & 11 & 7 & 8 & 6 & 6 & 96 \\
\hline $\begin{array}{l}\text { Journal of Hospitality } \\
\text { and Tourism Research }\end{array}$ & 2 & 1 & 6 & 1 & & 1 & 1 & 1 & 2 & 6 & 2 & & 23 \\
\hline Journal of Travel Research & 1 & 4 & & & 1 & 1 & 1 & 1 & & 2 & 1 & 1 & 13 \\
\hline Tourism Management & 3 & 2 & 1 & 4 & 3 & 1 & 3 & 5 & 6 & 4 & 10 & 2 & 44 \\
\hline Total & 10 & 11 & 15 & 14 & 18 & 15 & 14 & 18 & 15 & 20 & 19 & 9 & 178 \\
\hline
\end{tabular}




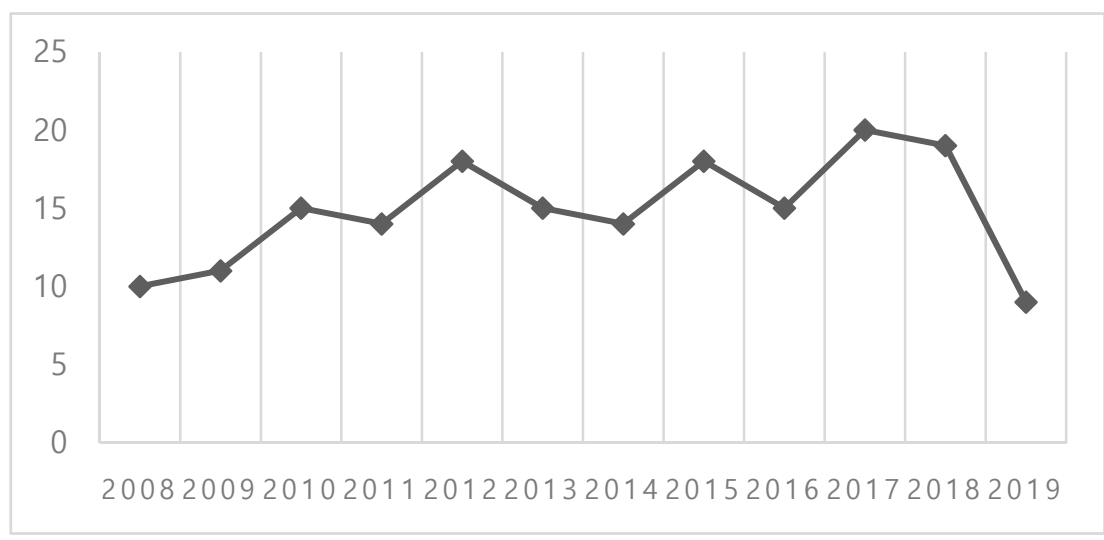

Figure 1. Quantity of publications per year (2008-2019).

Table 2 lists the journal, year, title, and author keywords associated with 178 research paper used in this research analysis. Using Excel, the data set was first arranged by journal name in ascending order. Then, the data set was arranged in ascending order by year and then title.

Table 2. Title and Author Keywords (2008-2019).

\begin{tabular}{|c|c|c|}
\hline \multirow{2}{*}{ No. } & \multicolumn{2}{|c|}{ Journals } \\
\hline & Title (Year) & Author Keywords \\
\hline \multicolumn{3}{|c|}{ Annals of Tourism Research } \\
\hline 1 & $\begin{array}{l}\text { Tourist experience and Wetland parks: A case of Zhejiang, } \\
\text { China (2012) }\end{array}$ & $\begin{array}{l}\text { China; Post-trip behavioral intention; service quality; } \\
\text { Tourist experience; Wetland parks }\end{array}$ \\
\hline 2 & Value Co-creation significance of tourist resources (2013) & $\begin{array}{l}\text { Effort; Involvement; Time spent; Tourist resources; } \\
\text { Value perception }\end{array}$ \\
\hline \multicolumn{3}{|c|}{ International Journal of Hospitality Management } \\
\hline 3 & $\begin{array}{l}\text { Customer value in the hotel industry: What managers } \\
\text { believe they deliver and what customer experience (2008) }\end{array}$ & $\begin{array}{l}\text { Business hotels; Customer value; Hospitality } \\
\text { industry; service quality }\end{array}$ \\
\hline 4 & $\begin{array}{l}\text { Determinants of dining satisfaction and post-dining } \\
\text { behavioral intentions (2008) }\end{array}$ & $\begin{array}{l}\text { Emotions; Loyalty; Recommendation; Restaurant } \\
\text { services; Satisfaction; service quality }\end{array}$ \\
\hline 5 & $\begin{array}{l}\text { Does bureaucracy kill individual initiative? The impact of } \\
\text { structure on organizational citizenship behavior in } \\
\text { the hospitality industry (2008) }\end{array}$ & $\begin{array}{l}\text { Bureaucracy; Centralization; Formalization; Hotel } \\
\text { industry; Organizational citizenship behavior; } \\
\text { service quality }\end{array}$ \\
\hline 6 & $\begin{array}{l}\text { The interaction of major resources and their influence on } \\
\text { waiting times in a multi-stage restaurant (2008) }\end{array}$ & $\begin{array}{l}\text { Capacity management; Restaurant resource; service } \\
\text { quality; Simulation; Waiting time }\end{array}$ \\
\hline 7 & $\begin{array}{l}\text { Customers' cognitive, emotional, and actionable response } \\
\text { to the servicescape: A test of the moderating effect of } \\
\text { the restaurant type (2009) }\end{array}$ & $\begin{array}{l}\text { Emotion; Perceived service quality; Revisit intention; } \\
\text { servicescape; Theme restaurant }\end{array}$ \\
\hline 8 & $\begin{array}{l}\text { From public to private: Has employment practice changed } \\
\text { in Bulgarian hotels? (2009) }\end{array}$ & $\begin{array}{l}\text { Bulgaria; Emerging economies; Employment policy } \\
\text { and practice; Hotels }\end{array}$ \\
\hline 9 & $\begin{array}{l}\text { Influence of institutional DINESERV on customer } \\
\text { satisfaction, return intention, and word-of-mouth (2009) }\end{array}$ & $\begin{array}{l}\text { Customer satisfaction; Food quality; Institutional } \\
\text { DINESERV; Return intention; Word-of-mouth } \\
\text { endorsement }\end{array}$ \\
\hline 10 & Tipping behavior in Canadian restaurants (2009) & $\begin{array}{l}\text { Canadian restaurant industry; CREST data; } \\
\text { Gratuities; Tipping }\end{array}$ \\
\hline 11 & $\begin{array}{l}\text { An investigation of green hotel customers' decision } \\
\text { formation: Developing an extended model of the theory of } \\
\text { planned behavior (2010) }\end{array}$ & $\begin{array}{l}\text { Decision-making; Extended theory of planned } \\
\text { behavior; Green hotel; Revisit intention }\end{array}$ \\
\hline 12 & $\begin{array}{l}\text { Applying loss aversion to assess the effect of customers' } \\
\text { asymmetric responses to service quality on post-dining } \\
\text { behavioral intentions: An empirical survey in } \\
\text { the restaurant sector (2010) }\end{array}$ & $\begin{array}{l}\text { Customers' post-behavioral intentions; DINESERV; } \\
\text { Loss aversion }\end{array}$ \\
\hline 13 & $\begin{array}{l}\text { Effects of organizational/occupational characteristics } \\
\text { and personality traits on hotel manager emotional } \\
\text { exhaustion (2010) }\end{array}$ & $\begin{array}{l}\text { Burnout; Emotional exhaustion; Hotel; } \\
\text { Personality traits }\end{array}$ \\
\hline 14 & $\begin{array}{l}\text { Effects of service quality and food quality: The moderating } \\
\text { role of atmospherics in an ethnic restaurant segment (2010) }\end{array}$ & $\begin{array}{l}\text { Atmospherics; Ethnic restaurant; Food quality; } \\
\text { Korean restaurant; Loyalty; Moderating effect; } \\
\text { Satisfaction; service quality }\end{array}$ \\
\hline 15 & $\begin{array}{l}\text { Exploratory assessment of the Macao casino dealers' job } \\
\text { perceptions (2010) }\end{array}$ & $\begin{array}{l}\text { Casino dealers; Front-line employees; Job } \\
\text { perceptions; Macao }\end{array}$ \\
\hline
\end{tabular}


Table 2. Cont

\begin{tabular}{|c|c|c|}
\hline \multirow{2}{*}{ No. } & \multicolumn{2}{|c|}{ Journals } \\
\hline & Title (Year) & Author Keywords \\
\hline \multicolumn{3}{|c|}{ International Journal of Hospitality Management } \\
\hline 16 & $\begin{array}{l}\text { Lifestyle businesses: Insights into Blackpool's hotel } \\
\text { sector (2010) }\end{array}$ & $\begin{array}{l}\text { Blackpool hotels; Lifestyle entrepreneurs; } \\
\text { Micro-businesses }\end{array}$ \\
\hline 17 & $\begin{array}{l}\text { Pay for performance and work attitudes: The mediating } \\
\text { role of employee-organization service value } \\
\text { congruence (2010) }\end{array}$ & $\begin{array}{l}\text { P-O fit; Pay for performance; service value } \\
\text { congruence; Work attitudes }\end{array}$ \\
\hline 18 & $\begin{array}{l}\text { Supply management practices and performance in } \\
\text { the Canadian hospitality industry (2010) }\end{array}$ & $\begin{array}{l}\text { Hospitality; Performance; Purchasing strategy; } \\
\text { service; Supply management }\end{array}$ \\
\hline 19 & $\begin{array}{l}\text { An examination of electronic video clips in the context of } \\
\text { hotel Websites (2011) }\end{array}$ & $\begin{array}{l}\text { E-VISQUAL; Experiential services; Video clip; Virtual } \\
\text { human interaction; Visual image }\end{array}$ \\
\hline 20 & $\begin{array}{l}\text { Customers' perceptions of service quality: Do servers' age } \\
\text { stereotypes matter? (2011) }\end{array}$ & $\begin{array}{l}\text { Age stereotypes; Perceptions of service quality; } \\
\text { Server }\end{array}$ \\
\hline 21 & $\begin{array}{l}\text { Foreign travelers' satisfaction with traditional Korean } \\
\text { restaurants (2011) }\end{array}$ & $\begin{array}{l}\text { Customer satisfaction; Expectation; Korean } \\
\text { restaurants; service quality; Value for money }\end{array}$ \\
\hline 22 & $\begin{array}{l}\text { Luxury marketing: The influences of psychological } \\
\text { and demographic characteristics on attitudes toward } \\
\text { luxury restaurants (2011) }\end{array}$ & $\begin{array}{l}\text { Demographics; Hedonism; Luxury marketing; } \\
\text { Materialism; Perfectionism; Uniqueness }\end{array}$ \\
\hline 23 & $\begin{array}{l}\text { Restaurant consumers repeat patronage: A service quality } \\
\text { concern (2011) }\end{array}$ & $\begin{array}{l}\text { Cleanliness; Consumers; Repeat patronage; } \\
\text { Restaurant; service quality }\end{array}$ \\
\hline 24 & $\begin{array}{l}\text { Restaurant experiences triggering positive electronic } \\
\text { word-of-mouth (eWOM) motivations (2011) }\end{array}$ & $\begin{array}{l}\text { Electronic word-of-mouth (eWOM); EWOM } \\
\text { motivations; Perceived service quality; } \\
\text { Restaurant experiences }\end{array}$ \\
\hline 25 & $\begin{array}{l}\text { Reward climate and its impact on service quality } \\
\text { orientation and employee attitudes (2011) }\end{array}$ & $\begin{array}{l}\text { Job satisfaction; Organizational commitment; } \\
\text { Reward climate; service quality orientation (SQO) }\end{array}$ \\
\hline 26 & $\begin{array}{l}\text { Strategic alignment leverage between hotels } \\
\text { and companies: The buyer-supplier relationship } \\
\text { perspective (2011) }\end{array}$ & $\begin{array}{l}\text { Environment uncertainty; service vision; } \\
\text { Strategic alignment }\end{array}$ \\
\hline 27 & $\begin{array}{l}\text { When will customers care about service failures that } \\
\text { happened to strangers? The role of personal similarity } \\
\text { and regulatory focus and its implication on service } \\
\text { evaluation (2011) }\end{array}$ & $\begin{array}{l}\text { Attribution; Personal similarity; Regulatory focus; } \\
\text { service failures }\end{array}$ \\
\hline 28 & $\begin{array}{l}\text { Customer opportunistic complaints management: } \\
\text { A critical incident approach (2012) }\end{array}$ & $\begin{array}{l}\text { Complaining behavior; Critical incident technique; } \\
\text { Opportunistic complaint; Unethical consumer } \\
\text { behavior }\end{array}$ \\
\hline 29 & $\begin{array}{l}\text { Development and validation of the casino service quality } \\
\text { scale: CASERV (2012) }\end{array}$ & Casino; Macau; Scale development; service quality \\
\hline 30 & $\begin{array}{l}\text { Does better service induce higher profitability? Evidence } \\
\text { from Taiwanese Hospitality Industry (2012) }\end{array}$ & $\begin{array}{l}\text { International tourist hotel; Profitability; service } \\
\text { quality }\end{array}$ \\
\hline 31 & $\begin{array}{l}\text { Enhancing service quality improvement strategies of } \\
\text { fine-dining restaurants: New insights from integrating } \\
\text { a two-phase decision-making model of } \\
\text { IPGA and DEMATEL analysis (2012) }\end{array}$ & DEMATEL; IPGA; Restaurant service quality \\
\hline 32 & Factorial validation of hospitality service attitude (2012) & $\begin{array}{l}\text { Customer satisfaction; Hotel industry; Interaction } \\
\text { quality; service attitude; service quality }\end{array}$ \\
\hline 33 & $\begin{array}{l}\text { It is all about the emotional state: Managing tourists' } \\
\text { experiences (2012) }\end{array}$ & $\begin{array}{l}\text { Customer satisfaction; Emotional state; Hotel setting; } \\
\text { Leisure experience; servicescape }\end{array}$ \\
\hline 34 & $\begin{array}{l}\text { Measurement scale for eco-component of hotel service } \\
\text { quality (2012) }\end{array}$ & $\begin{array}{l}\text { Eco-component; Eco-label; Ecological expectations; } \\
\text { Hotel service quality; Wellness hotels }\end{array}$ \\
\hline 35 & $\begin{array}{l}\text { Relationship quality between exhibitors and organizers: } \\
\text { A perspective from Mainland China's exhibition } \\
\text { industry (2012) }\end{array}$ & China; Exhibitions; Relationship quality \\
\hline 36 & $\begin{array}{l}\text { service guarantees in the hotel industry: Their effects on } \\
\text { consumer risk and service quality perceptions (2012) }\end{array}$ & $\begin{array}{l}\text { Corporate reputation; Hotel; Perceived quality; } \\
\text { Perceived risk; service guarantees }\end{array}$ \\
\hline 37 & $\begin{array}{l}\text { service quality and tipping: The moderating role of } \\
\text { the quality of food (2012) }\end{array}$ & Quality of food; service quality; Tipping behavior \\
\hline 38 & $\begin{array}{l}\text { service-leadership competencies for hospitality } \\
\text { and tourism management (2012) }\end{array}$ & $\begin{array}{l}\text { Competency model; Hospitality and tourism; } \\
\text { Leadership; service quality }\end{array}$ \\
\hline 39 & $\begin{array}{l}\text { The underlying dimensions of tipping behavior: } \\
\text { An exploration, confirmation, and predictive model (2012) }\end{array}$ & $\begin{array}{l}\text { Consumer behavior; Motivation; Restaurants; service } \\
\text { quality; Social norms; Tipping }\end{array}$ \\
\hline 40 & $\begin{array}{l}\text { When I put on my service mask: Determinants } \\
\text { and outcomes of emotional labor among hotel service } \\
\text { providers according to affective event theory (2012) }\end{array}$ & $\begin{array}{l}\text { Affective event theory; Emotional labor; Hotel service } \\
\text { employees; Negative emotions; service quality }\end{array}$ \\
\hline 41 & $\begin{array}{l}\text { Corporate social responsibility practices in four } \\
\text { and five-star hotels: Perspectives from Hong Kong } \\
\text { visitors (2013) }\end{array}$ & $\begin{array}{l}\text { Corporate social responsibility; } \\
\text { Customer perceptions; Hong Kong; Hotels }\end{array}$ \\
\hline
\end{tabular}


Table 2. Cont.

\begin{tabular}{|c|c|c|}
\hline \multirow{2}{*}{ No. } & \multicolumn{2}{|c|}{ Journals } \\
\hline & Title (Year) & Author Keywords \\
\hline \multicolumn{3}{|c|}{ International Journal of Hospitality Management } \\
\hline 42 & $\begin{array}{l}\text { Emotional intelligence and adaptability-service encounters } \\
\text { between casino hosts and premium players (2013) }\end{array}$ & $\begin{array}{l}\text { Adaptability; Casino hosts; Casino industry; } \\
\text { Emotional intelligence; Premium player; service } \\
\text { performance }\end{array}$ \\
\hline 43 & $\begin{array}{l}\text { Factors influencing internal service quality at international } \\
\text { tourist hotels (2013) }\end{array}$ & $\begin{array}{l}\text { Hospitality service; Internal service quality; } \\
\text { Leadership; Organizational culture }\end{array}$ \\
\hline 44 & $\begin{array}{l}\text { Factors influencing the effectiveness of online group } \\
\text { buying in the restaurant industry (2013) }\end{array}$ & $\begin{array}{l}\text { Discount; Group buying; Restaurant; } \\
\text { Return intention; Satisfaction; service quality }\end{array}$ \\
\hline 45 & $\begin{array}{l}\text { Is satisfaction enough to ensure reciprocity with upscale } \\
\text { restaurants? The role of gratitude relative to } \\
\text { satisfaction (2013) }\end{array}$ & $\begin{array}{l}\text { Gratitude; Reciprocal behaviors; Relationship } \\
\text { benefits; Satisfaction; Upscale restaurant }\end{array}$ \\
\hline 46 & $\begin{array}{l}\text { Justice perceptions and drives of hotel employee social } \\
\text { loafing behavior (2013) }\end{array}$ & $\begin{array}{l}\text { China hotel industry; Commitment; Justice } \\
\text { perceptions; Social loafing; Turnover intention }\end{array}$ \\
\hline 47 & $\begin{array}{l}\text { Multi-dimensions of patrons' emotional experiences in } \\
\text { upscale restaurants and their role in loyalty formation: } \\
\text { Emotion scale improvement (2013) }\end{array}$ & $\begin{array}{l}\text { Commitment; Emotion measurement; Image; Loyalty } \\
\text { intentions; Satisfaction; service quality; Trust; } \\
\text { Upscale restaurant }\end{array}$ \\
\hline 48 & $\begin{array}{l}\text { The effect of regulatory focus and delay type on } \\
\text { consumers' reactions to delay (2013) }\end{array}$ & $\begin{array}{l}\text { Affective response; Delay type; Expectancy model; } \\
\text { Field theory; Regulatory focus; service quality }\end{array}$ \\
\hline 49 & $\begin{array}{l}\text { The effects of restaurant servers' perceptions of customers' } \\
\text { tipping behaviors on service discrimination (2013) }\end{array}$ & $\begin{array}{l}\text { Buyer monitoring; Employee control mechanisms; } \\
\text { Restaurant; service discrimination; service quality; } \\
\text { Tipping }\end{array}$ \\
\hline 50 & $\begin{array}{l}\text { The influence of customer brand identification on hotel } \\
\text { brand evaluation and loyalty development (2013) }\end{array}$ & $\begin{array}{l}\text { Brand loyalty; Brand trust; Identification; Perceived } \\
\text { value; service quality }\end{array}$ \\
\hline 51 & $\begin{array}{l}\text { The role of frequency of patronage and service quality of } \\
\text { all-you-can-eat buffet restaurant: A perspective of } \\
\text { socio-economic and demographic characteristics of African } \\
\text { American consumers (2013) }\end{array}$ & $\begin{array}{l}\text { African American consumers; Blacks; Buffet } \\
\text { restaurant marketing; Food consumption; Restaurant } \\
\text { patronage; service quality }\end{array}$ \\
\hline 52 & $\begin{array}{l}\text { A patron, a referral and why in Macau casinos -The case of } \\
\text { mainland Chinese gamblers (2014) }\end{array}$ & $\begin{array}{l}\text { Attitudinal loyalty; Behavioural loyalty; Casino } \\
\text { service quality; Chinese gamblers; Loyalty } \\
\text { programme; Macau casinos; Membership }\end{array}$ \\
\hline 53 & $\begin{array}{l}\text { Applying service Profit Chain model to the Korean } \\
\text { restaurant industry (2014) }\end{array}$ & $\begin{array}{l}\text { Customer constructs; Employee constructs; Internal } \\
\text { service quality; Korean restaurant; Organizational } \\
\text { commitment; service Profit Chain }\end{array}$ \\
\hline 54 & $\begin{array}{l}\text { Attitudinal dimensions of professionalism and service } \\
\text { quality efficacy of frontline employees in hotels (2014) }\end{array}$ & $\begin{array}{l}\text { Customer orientation; Knowledge; Professionalism; } \\
\text { Self-efficacy; Self-management; Sense of calling }\end{array}$ \\
\hline 55 & $\begin{array}{l}\text { Becoming an ambidextrous hotel: The role of customer } \\
\text { orientation (2014) }\end{array}$ & $\begin{array}{l}\text { Customer orientation; Exploitation; Exploration; } \\
\text { service improvement; service innovation }\end{array}$ \\
\hline 56 & $\begin{array}{l}\text { Effective restaurant rating scale development } \\
\text { and a mystery shopper evaluation approach (2014) }\end{array}$ & $\begin{array}{l}\text { Mystery shopper; Restaurant; Restaurant rating; } \\
\text { Scale development; service quality }\end{array}$ \\
\hline 57 & $\begin{array}{l}\text { Job standardization and service quality: The mediating } \\
\text { role of prosocial service behaviors (2014) }\end{array}$ & $\begin{array}{l}\text { Job standardization; Prosocial service behaviors; } \\
\text { service quality }\end{array}$ \\
\hline 58 & $\begin{array}{l}\text { Linking service quality, customer satisfaction and loyalty } \\
\text { in casinos, does membership matter? (2014) }\end{array}$ & $\begin{array}{l}\text { Casino marketing, Macau; Customer loyalty; } \\
\text { Customer satisfaction; Membership; service quality }\end{array}$ \\
\hline 59 & $\begin{array}{l}\text { Why do customers switch? More satiated or less } \\
\text { satisfied (2014) }\end{array}$ & $\begin{array}{l}\text { Atmospheric quality; Food quality; Satiation; } \\
\text { Satisfaction; service quality; Switching intention }\end{array}$ \\
\hline 60 & $\begin{array}{l}\text { Wine attributes, perceived risk and online wine repurchase } \\
\text { intention: The cross-level interaction effects of website } \\
\text { quality (2014) }\end{array}$ & $\begin{array}{l}\text { Hierarchical linear modeling; Multilevel analysis; } \\
\text { Online wine repurchase intention; Perceived risk; } \\
\text { Website quality; Wine attributes }\end{array}$ \\
\hline 61 & $\begin{array}{l}\text { A conflict of choice: How consumers choose where to go } \\
\text { for dinner (2015) }\end{array}$ & $\begin{array}{l}\text { Food quality; Multi-attribute decision making; } \\
\text { Restaurant choice experiment; service quality }\end{array}$ \\
\hline 62 & $\begin{array}{l}\text { A segmentation of online reviews by language groups: } \\
\text { How English and non-English speakers rate hotels } \\
\text { differently (2015) }\end{array}$ & $\begin{array}{l}\text { Language; Online ratings; Reputation; } \\
\text { Satisfaction difference; Traveler distribution }\end{array}$ \\
\hline 63 & $\begin{array}{l}\text { Antecedents of mandatory customer participation in } \\
\text { service encounters: An empirical study (2015) }\end{array}$ & $\begin{array}{l}\text { Customer participation; Loyalty; Purchase } \\
\text { importance; Role clarity; Self-efficacy; service quality }\end{array}$ \\
\hline 64 & $\begin{array}{l}\text { Applying mixed methods to identify what drives quick } \\
\text { service restaurant's customer satisfaction } \\
\text { at the unit-level (2015) }\end{array}$ & $\begin{array}{l}\text { Customer satisfaction; Kano's model; Performance } \\
\text { optimization; Product quality; Quick service } \\
\text { restaurants; service quality }\end{array}$ \\
\hline 65 & $\begin{array}{l}\text { Consumer-based chain restaurant brand equity, brand } \\
\text { reputation, and brand trust (2015) }\end{array}$ & $\begin{array}{l}\text { Brand equity; Brand reputation; Brand trust; } \\
\text { Chain restaurant; Visit purpose }\end{array}$ \\
\hline 66 & 5) & $\begin{array}{l}\text { Capital-labor ratio; Hotel industry; service quality; } \\
\text { Uncertain demand }\end{array}$ \\
\hline 67 & $\begin{array}{l}\text { Impacts of cruise service quality and price on vacationers' } \\
\text { cruise experience: Moderating role of price } \\
\text { sensitivity (2015) }\end{array}$ & $\begin{array}{l}\text { Cruise; Loyalty; Novelty; Price; Price sensitivity; } \\
\text { service quality; Value }\end{array}$ \\
\hline
\end{tabular}


Table 2. Cont

\begin{tabular}{|c|c|c|}
\hline \multirow{2}{*}{ No. } & \multicolumn{2}{|c|}{ Journals } \\
\hline & Title (Year) & Author Keywords \\
\hline \multicolumn{3}{|c|}{ International Journal of Hospitality Management } \\
\hline 68 & $\begin{array}{l}\text { Positive emotions and behavioral intentions of customers } \\
\text { in full-service restaurants: Does aesthetic labor } \\
\text { matter? (2015) }\end{array}$ & $\begin{array}{l}\text { Aesthetic labor; Behavioral intention; Full-service } \\
\text { restaurant; Positive emotion }\end{array}$ \\
\hline 69 & $\begin{array}{l}\text { The cross-impact of network externalities on relationship } \\
\text { quality in exhibition sector (2015) }\end{array}$ & $\begin{array}{l}\text { Customer loyalty; Exhibition; Network externalities; } \\
\text { Relationship quality; service quality }\end{array}$ \\
\hline 70 & $\begin{array}{l}\text { Understanding service experience and its impact on brand } \\
\text { image in hospitality sector (2015) }\end{array}$ & $\begin{array}{l}\text { Brand image; Customer experiences; Customer } \\
\text { Satisfaction; India; service quality }\end{array}$ \\
\hline 71 & $\begin{array}{l}\text { Value dimensions in consumers' experience: Combining } \\
\text { the intra- and inter-variable approaches in the hospitality } \\
\text { sector (2015) }\end{array}$ & $\begin{array}{l}\text { Extrinsic vs. intrinsic values; Loyalty; } \\
\text { Perceived value; Satisfaction; Value dimensions }\end{array}$ \\
\hline 72 & $\begin{array}{l}\text { Adaptation of hospitality service quality scales for marina } \\
\text { services (2016) }\end{array}$ & $\begin{array}{l}\text { Marinas; service quality; Turkish marinas; } \\
\text { Yachtsmen's satisfaction }\end{array}$ \\
\hline 73 & $\begin{array}{l}\text { An emotional labor perspective on the relationship } \\
\text { between customer orientation and job satisfaction (2016) }\end{array}$ & $\begin{array}{l}\text { Customer orientation; Emotional labor; } \\
\text { Job satisfaction }\end{array}$ \\
\hline 74 & $\begin{array}{l}\text { Do competitive strategy effects vary across hotel industry } \\
\text { cycles? (2016) }\end{array}$ & $\begin{array}{l}\text { Advertising expenditure; Business cycle; Hotel } \\
\text { industry; Pricing strategy; Quality strategy }\end{array}$ \\
\hline 75 & $\begin{array}{l}\text { Embracing or fighting the urge: A multilevel investigation } \\
\text { on casino service, branding and impulsive gambling (2016) }\end{array}$ & $\begin{array}{l}\text { Brand attachment; Brand equity; Casinos; Impulsive } \\
\text { gambling; Loyalty; service quality }\end{array}$ \\
\hline 76 & $\begin{array}{l}\text { Hedonic adaptation and satiation: Understanding } \\
\text { switching behavior in the restaurant industry (2016) }\end{array}$ & $\begin{array}{l}\text { Hedonic adaptation; Restaurants; Satiation; } \\
\text { Satisfaction; Switching }\end{array}$ \\
\hline 77 & $\begin{array}{l}\text { Internal branding process: Exploring the role of mediators } \\
\text { in top management's leadership-commitment } \\
\text { relationship (2016) }\end{array}$ & $\begin{array}{l}\text { Brand commitment; Employee brand knowledge; } \\
\text { Employee-brand fit; Psychological contract; } \\
\text { Top management's brand-oriented leadership }\end{array}$ \\
\hline 78 & $\begin{array}{l}\text { The influences of restaurant menu font style, background } \\
\text { color, and physical weight on consumers' } \\
\text { perceptions (2016) }\end{array}$ & $\begin{array}{l}\text { Menu background color; Menu font style; } \\
\text { Menu weight; Restaurant scale; Restaurant service }\end{array}$ \\
\hline 79 & $\begin{array}{l}\text { Exploring the nonlinear impact of critical incidents on } \\
\text { customers? general evaluation of hospitality } \\
\text { services (2017) }\end{array}$ & $\begin{array}{l}\text { Critical incident technique; Hospitality industry; } \\
\text { Kano model; service quality; TripAdvisor }\end{array}$ \\
\hline 80 & $\begin{array}{l}\text { Extending the scope of hotel client reactions to employee } \\
\text { injustice: Hotel employer reviews on the Internet (2017) }\end{array}$ & $\begin{array}{l}\text { Client satisfaction; Hotel employee (mis)treatment; } \\
\text { Hotel management; Organizational justice; } \\
\text { The Internet }\end{array}$ \\
\hline 81 & $\begin{array}{l}\text { Impact of hotel-restaurant image and quality of } \\
\text { physical-environment, service, and food on satisfaction } \\
\text { and intention (2017) }\end{array}$ & $\begin{array}{l}\text { Customer satisfaction; Food quality; Intentions; } \\
\text { Luxury hotel restaurant; Physical environment } \\
\text { quality; service quality }\end{array}$ \\
\hline 82 & $\begin{array}{l}\text { The effects of teppanyaki restaurant stimuli on diners? } \\
\text { Emotions and loyalty (2017) }\end{array}$ & Chef's image; Diners; Emotions; Loyalty; Teppanyaki \\
\hline 83 & $\begin{array}{l}\text { The impact of personal and functional aspects of } \\
\text { restaurant employee service behaviour on customer } \\
\text { satisfaction (2017) }\end{array}$ & $\begin{array}{l}\text { Customer satisfaction; Personal aspects; Restaurant } \\
\text { service quality; service behaviour; service encounter }\end{array}$ \\
\hline 84 & $\begin{array}{l}\text { The impact of the social servicescape, density, } \\
\text { and restaurant type on perceptions of interpersonal service } \\
\text { quality (2017) }\end{array}$ & $\begin{array}{l}\text { Density; DINESERV; Restaurant; service quality; } \\
\text { Social servicescape }\end{array}$ \\
\hline 85 & $\begin{array}{l}\text { Understanding the dimensions of customer relationships } \\
\text { in the hotel and restaurant industries (2017) }\end{array}$ & $\begin{array}{l}\text { Customer lifetime financial value; Customer } \\
\text { relationships; Hospitality marketing strategy; } \\
\text { Relationship marketing }\end{array}$ \\
\hline 86 & $\begin{array}{l}\text { Willingness to pay in negative restaurant service } \\
\text { encounters (2017) }\end{array}$ & $\begin{array}{l}\text { Ambiance; Food quality; service encounter; service } \\
\text { quality; Value; Willingness to pay }\end{array}$ \\
\hline 87 & $\begin{array}{l}\text { Projecting service quality: The effects of social media } \\
\text { reviews on service perception (2018) }\end{array}$ & $\begin{array}{l}\text { Social media reviews; Social media popularity; } \\
\text { Service quality; Credibility; Service attributes; } \\
\text { Facebook }\end{array}$ \\
\hline 88 & $\begin{array}{l}\text { Less is more: A new insight for measuring service quality } \\
\text { of green hotels (2018) }\end{array}$ & $\begin{array}{l}\text { and GLSERV scale; Carbon reduction; Environmental } \\
\text { protection; Green hotel; service quality }\end{array}$ \\
\hline 89 & $\begin{array}{l}\text { On the relationship between intellectual capital } \\
\text { and financial performance: A panel data analysis on SME } \\
\text { hotels (2018) }\end{array}$ & $\begin{array}{l}\text { Dynamic panel data; Financial performance; } \\
\text { Intellectual capital; Small and medium-sized } \\
\text { enterprise hotels }\end{array}$ \\
\hline 90 & $\begin{array}{l}\text { Projecting service quality: The effects of social media } \\
\text { reviews on service perception (2018) }\end{array}$ & $\begin{array}{l}\text { Credibility; Facebook; service attributes; service } \\
\text { quality; Social media popularity; Social media } \\
\text { reviews }\end{array}$ \\
\hline 91 & $\begin{array}{l}\text { The effects of organizational and personal resources on } \\
\text { stress, engagement, and job outcomes (2018) }\end{array}$ & $\begin{array}{l}\text { Customer orientation; Engagement; Hotel employees; } \\
\text { Job outcomes; Job stress; Management commitment } \\
\text { to service quality }\end{array}$ \\
\hline 92 & $\begin{array}{l}\text { The Integration between service Value and service } \\
\text { Recovery in the Hospitality Industry: An Application of } \\
\text { QFD and ANP (2018) }\end{array}$ & $\begin{array}{l}\text { Analytic network process; Customer satisfaction; } \\
\text { Quality function deployment; service failure; } \\
\text { service recovery }\end{array}$ \\
\hline
\end{tabular}


Table 2. Cont

\begin{tabular}{|c|c|c|}
\hline \multirow{2}{*}{ No. } & \multicolumn{2}{|c|}{ Journals } \\
\hline & Title (Year) & Author Keywords \\
\hline \multicolumn{3}{|c|}{ International Journal of Hospitality Management } \\
\hline 93 & $\begin{array}{l}\text { Changing tires on a moving car: the role of timing in } \\
\text { hospitality and service turnaround processes (2019) }\end{array}$ & $\begin{array}{l}\text { Hospitality industry; Recovery; Retrenchment; } \\
\text { service industry; Turnaround process }\end{array}$ \\
\hline 94 & $\begin{array}{l}\text { Consumer values and service quality perceptions of food } \\
\text { truck experiences (2019) }\end{array}$ & $\begin{array}{l}\text { DINESERV; Food truck; Hedonic value; } \\
\text { Importance-performance analysis; Utilitarian value }\end{array}$ \\
\hline 95 & $\begin{array}{l}\text { Developing and validating a mobile catering app success } \\
\text { model (2019) }\end{array}$ & $\begin{array}{l}\text { E-commerce system success model; eWOM; Mobile } \\
\text { catering app; Perceived price; Perceived promotions; } \\
\text { Product quality }\end{array}$ \\
\hline 96 & $\begin{array}{l}\text { Exploring Airbnb service quality attributes and their } \\
\text { asymmetric effects on customer satisfaction (2019) }\end{array}$ & $\begin{array}{l}\text { Airbnb; Customer satisfaction; Impact asymmetry } \\
\text { analysis; Impact range performance analysis; Mixed } \\
\text { method; service quality }\end{array}$ \\
\hline 97 & $\begin{array}{l}\text { Good discounts earn good reviews in return? Effects of } \\
\text { price promotion on online restaurant reviews (2019) }\end{array}$ & $\begin{array}{l}\text { Image; Online consumer review; Price promotion; } \\
\text { Restaurant; Review rating; Textual content }\end{array}$ \\
\hline 98 & $\begin{array}{l}\text { The impact of green practices in coastal tourism: } \\
\text { An empirical investigation on an eco-labelled beach } \\
\text { club (2019) }\end{array}$ & $\begin{array}{l}\text { Beach club; Behavioral intention; Coastal tourism; } \\
\text { Ecolabel; Green practices; PLS-SEM }\end{array}$ \\
\hline \multicolumn{3}{|c|}{ Journal of Hospitality and Tourism Research } \\
\hline 99 & $\begin{array}{l}\text { Relationships Among Experiential Marketing, Experiential } \\
\text { Value, and Customer Satisfaction (2008) }\end{array}$ & $\begin{array}{l}\text { customer satisfaction; experiential marketing; } \\
\text { experiential value; structural equation modeling }\end{array}$ \\
\hline 100 & $\begin{array}{l}\text { Tourists' Satisfaction Judgments: An Investigation of } \\
\text { Emotion, Equity, and Attribution (2008) }\end{array}$ & $\begin{array}{l}\text { attribution; culture; emotion; equity; package tour; } \\
\text { satisfaction }\end{array}$ \\
\hline 101 & $\begin{array}{l}\text { Stakeholder service perspectives: A triadic analysis of } \\
\text { service quality in south Mississippi fine dining } \\
\text { restaurants (2009) }\end{array}$ & Quality perceptions; service quality; Stakeholders \\
\hline 102 & $\begin{array}{l}\text { Does cultural difference affect customer's response in } \\
\text { a crowded restaurant environment? A comparison of } \\
\text { American versus Chinese customers (2010) }\end{array}$ & Attribution; Crowdedness; Cultural differences \\
\hline 103 & $\begin{array}{l}\text { Influence of the Quality of Food, service, and Physical } \\
\text { Environment on Customer Satisfaction and Behavioral } \\
\text { Intention in Quick-Casual Restaurants: Moderating Role } \\
\text { of Perceived Price (2010) }\end{array}$ & $\begin{array}{l}\text { behavioral intention; perceived price; quality } \\
\text { dimensions (food service and physical environment); } \\
\text { quick-casual restaurants; satisfaction }\end{array}$ \\
\hline 104 & $\begin{array}{l}\text { The role and effect of job satisfaction and empowerment on } \\
\text { customers' perception of service quality: A study in } \\
\text { the restaurant industry (2010) }\end{array}$ & $\begin{array}{l}\text { Customer satisfaction; Employee empowerment; } \\
\text { Employee job satisfaction; HRM-service quality link; } \\
\text { Restaurant services management; service quality }\end{array}$ \\
\hline 105 & $\begin{array}{l}\text { Tipping and service quality: A within-subjects } \\
\text { analysis (2010) }\end{array}$ & Equity; Incentive; Reward; service quality; Tipping \\
\hline 106 & $\begin{array}{l}\text { Tour guide performance and tourist satisfaction: A study } \\
\text { of the package tours in Shanghai (2010) }\end{array}$ & $\begin{array}{l}\text { service quality; Tour experience; Tour guide } \\
\text { performance; Tour operator; Tourist satisfaction }\end{array}$ \\
\hline 107 & $\begin{array}{l}\text { Toward a Theory of Restaurant Décor: An Empirical } \\
\text { Examination of Italian Restaurants in Manhattan }\end{array}$ & $\begin{array}{l}\text { New York restaurants; restaurant décor; theory of } \\
\text { reasoned action }\end{array}$ \\
\hline 108 & $\begin{array}{l}\text { Synthesizing the effects of service quality, value, } \\
\text { and customer satisfaction on behavioral intentions in } \\
\text { the motel industry: An empirical analysis (2011) }\end{array}$ & $\begin{array}{l}\text { behavioral intentions; customer satisfaction; motel } \\
\text { industry; service quality; value }\end{array}$ \\
\hline 109 & $\begin{array}{l}\text { An Exploratory Examination of Supervisor Undermining, } \\
\text { Employee Involvement Climate, and the Effects on } \\
\text { Customer Perceptions of service quality in Quick-service } \\
\text { Restaurants (2013) }\end{array}$ & $\begin{array}{l}\text { customer service; employee involvement; } \\
\text { quick-service restaurants; undermining }\end{array}$ \\
\hline 110 & $\begin{array}{l}\text { The Influence of Hotel Price on Perceived service quality } \\
\text { and Value in E-Tourism: An Empirical Investigation Based } \\
\text { on Online Traveler Reviews (2014) }\end{array}$ & $\begin{array}{l}\text { online reviews; perceived quality; perceived value; } \\
\text { price effects }\end{array}$ \\
\hline 111 & Enhancing Consumer Value in Wine Tourism (2015) & $\begin{array}{l}\text { consumption values; experiential marketing; } \\
\text { marketing; service quality }\end{array}$ \\
\hline 112 & $\begin{array}{l}\text { A quality-Value-Attitude Model: The Case of Expo } 2010 \\
\text { Shanghai (2016) }\end{array}$ & $\begin{array}{l}\text { attitude; economic value; emotional value; } \\
\text { mega-event quality; Shanghai Expo } \\
\text { customer expectations; dimensions of social }\end{array}$ \\
\hline 113 & $\begin{array}{l}\text { Socially Responsible Customers and the Evaluation of } \\
\text { service quality (2016) }\end{array}$ & $\begin{array}{l}\text { responsibility; ethical consumers; service quality; } \\
\text { social responsibility dimension scale; socially } \\
\text { responsible consumers }\end{array}$ \\
\hline 114 & $\begin{array}{l}\text { Construction and Validation of the Customer Participation } \\
\text { Scale (2017) }\end{array}$ & $\begin{array}{l}\text { customer loyalty; customer roles; mandatory } \\
\text { customer participation; scale development; } \\
\text { service quality }\end{array}$ \\
\hline 115 & $\begin{array}{l}\text { Signaling service quality via Website e-CRM Features: } \\
\text { More Gains for Smaller and Lesser Known Hotels }\end{array}$ & $\begin{array}{l}\text { Signaling theory; electronic customer relationship } \\
\text { management; e-CRM, website quality }\end{array}$ \\
\hline
\end{tabular}


Table 2. Cont

\begin{tabular}{|c|c|c|}
\hline \multirow{2}{*}{ No. } & \multicolumn{2}{|c|}{ Journals } \\
\hline & Title (Year) & Author Keywords \\
\hline \multicolumn{3}{|c|}{ Journal of Hospitality and Tourism Research } \\
\hline 116 & $\begin{array}{l}\text { Examination of Restaurant Quality, Relationship Benefits, } \\
\text { and Customer Reciprocity from the Perspective of } \\
\text { Relationship Marketing Investments (2017) }\end{array}$ & $\begin{array}{l}\text { customer reciprocity; relationship benefit; } \\
\text { relationship marketing investment; restaurant quality }\end{array}$ \\
\hline 117 & $\begin{array}{l}\text { Psychological Capital in the Quick Service Restaurant } \\
\text { Industry: A Study of Unit-Level Performance (2017) }\end{array}$ & $\begin{array}{l}\text { customer satisfaction; psychological capital; quick } \\
\text { service restaurants; revenues; service quality }\end{array}$ \\
\hline 118 & $\begin{array}{l}\text { Signaling service quality via Website e-CRM Features: } \\
\text { More Gains for Smaller and Lesser Known Hotels (2017) }\end{array}$ & $\begin{array}{l}\text { e-CRM; electronic customer relationship } \\
\text { management: signaling theory; website quality }\end{array}$ \\
\hline & The Effect of Tourist Relationship Perception on & \\
\hline 119 & $\begin{array}{l}\text { Destination Loyalty at a World Heritage Site in China: } \\
\text { The Mediating Role of Overall Destination Satisfaction } \\
\text { and Trust (2017) }\end{array}$ & $\begin{array}{l}\text { destination image; loyalty; satisfaction; service } \\
\text { fairness; service quality; trust }\end{array}$ \\
\hline 120 & $\begin{array}{l}\text { The Effect of Event Supportive Service Environment } \\
\text { and Authenticity in the Quality-Value-Satisfaction } \\
\text { Framework (2018) }\end{array}$ & $\begin{array}{l}\text { authenticity; customer satisfaction; festival; perceived } \\
\text { value; program quality; service environment }\end{array}$ \\
\hline 121 & $\begin{array}{l}\text { Tourist Shoppers??Evaluation of Retail service: A Study of } \\
\text { Cross-Border Versus International Outshoppers (2018) }\end{array}$ & $\begin{array}{l}\text { cross-border outshoppers; evaluation of retail service; } \\
\text { international outshoppers; tourist shopping behavior }\end{array}$ \\
\hline \multicolumn{3}{|c|}{ Journal of Travel Research } \\
\hline 122 & $\begin{array}{l}\text { The effects of quality and satisfaction on awareness } \\
\text { and behavioral intentions: Exploring the role of a wine } \\
\text { festival (2008) }\end{array}$ & $\begin{array}{l}\text { Awareness; Behavioral intentions; Perceived quality; } \\
\text { Satisfaction; Wine festival }\end{array}$ \\
\hline 123 & $\begin{array}{l}\text { "You felt like lingering": Experiencing "real" service } \\
\text { at the winery tasting room (2009) }\end{array}$ & $\begin{array}{l}\text { Hospitality; service experience; service quality; Wine } \\
\text { tourism }\end{array}$ \\
\hline 124 & $\begin{array}{l}\text { A mediation model of tourists' repurchase intentions for } \\
\text { packaged tour services (2009) }\end{array}$ & $\begin{array}{l}\text { Packaged tour services; Perceived value; Repurchase } \\
\text { intentions; Satisfaction; service quality }\end{array}$ \\
\hline 125 & $\begin{array}{l}\text { Small-business owners' knowledge and rural tourism } \\
\text { establishment performance in Spain (2009) }\end{array}$ & $\begin{array}{l}\text { Knowledge; Objective quality; Perceived quality; } \\
\text { Rural tourism; Small-business owner }\end{array}$ \\
\hline 126 & $\begin{array}{l}\text { Tip-Collection strategies, service guarantees, } \\
\text { and consumer evaluations of group package tours (2009) }\end{array}$ & $\begin{array}{l}\text { Consumer evaluations; Group package tours; service } \\
\text { guarantee; Tip-collection strategy }\end{array}$ \\
\hline 127 & $\begin{array}{l}\text { The role of cybermediaries in reputation building } \\
\text { and price premiums in the online hotel market }(2012)\end{array}$ & $\begin{array}{l}\text { cybermediary; online hotel market; online travel } \\
\text { agent; reputation }\end{array}$ \\
\hline 128 & $\begin{array}{l}\text { A Systematic Approach to Scale Development in Tourist } \\
\text { Shopping Satisfaction: Linking Destination Attributes } \\
\text { and Shopping Experience (2013) }\end{array}$ & $\begin{array}{l}\text { experience; satisfaction; scale development; tourism } \\
\text { shopping; tourist facility }\end{array}$ \\
\hline 129 & $\begin{array}{l}\text { Guests and Hosts Revisited: Prejudicial Attitudes of } \\
\text { Guests toward the Host Population (2014) }\end{array}$ & $\begin{array}{l}\text { cultural bubble; host-guest relationships; prejudice; } \\
\text { tourism's impact; tourist attitudes }\end{array}$ \\
\hline 130 & $\begin{array}{l}\text { Perceived Destination Image: An Image Model for } \\
\text { a Winter Sports Destination and Its Effect on Intention to } \\
\text { Revisit (2015) }\end{array}$ & $\begin{array}{l}\text { destination image model; SEM; service quality; sport } \\
\text { events; visitor management }\end{array}$ \\
\hline 131 & $\begin{array}{l}\text { Enhancing service Loyalty: The Roles of Delight, } \\
\text { Satisfaction, and service quality (2017) }\end{array}$ & $\begin{array}{l}\text { delight; experience; loyalty; PLS path modeling; } \\
\text { quality; satisfaction }\end{array}$ \\
\hline 132 & $\begin{array}{l}\text { Service Quality Perceptions, Online Visibility, and Business } \\
\text { Performance in Rural Lodging Establishments (2017) }\end{array}$ & $\begin{array}{l}\text { business performance; online comments; qualitative } \\
\text { content analysis; rural tourism; web visibility }\end{array}$ \\
\hline 133 & $\begin{array}{l}\text { Medical Tourism Experience: Conceptualization, Scale } \\
\text { Development, and Validation (2018) }\end{array}$ & $\begin{array}{l}\text { health tourism; tourist perception; travel experience; } \\
\text { wellness tourism }\end{array}$ \\
\hline 134 & $\begin{array}{l}\text { Flying to Quality: Cultural Influences on Online } \\
\text { Reviews (2019) }\end{array}$ & $\begin{array}{l}\text { airlines; cultural differences; electronic WOM; online } \\
\text { reviews; service quality; structural topic model }\end{array}$ \\
\hline \multicolumn{3}{|c|}{ Tourism Management } \\
\hline 135 & $\begin{array}{l}\text { A service quality measurement architecture for hot spring } \\
\text { hotels in Taiwan (2008) }\end{array}$ & $\begin{array}{l}\text { Analysis network process (ANP); Hot spring hotels; } \\
\text { service quality }\end{array}$ \\
\hline 136 & $\begin{array}{l}\text { Developing a multidimensional and hierarchical service } \\
\text { quality model for the travel agency industry (2008) }\end{array}$ & $\begin{array}{l}\text { Multidimensional and hierarchical structure; service } \\
\text { quality; Travel agencies industry }\end{array}$ \\
\hline 137 & $\begin{array}{l}\text { Do airline self-service check-in kiosks meet the needs of } \\
\text { passengers? (2008) }\end{array}$ & $\begin{array}{l}\text { Importance-performance analysis; Ridit analysis; } \\
\text { Self-service kiosks }\end{array}$ \\
\hline 138 & $\begin{array}{l}\text { A multi-criteria assessment of tourist farm service } \\
\text { quality (2009) }\end{array}$ & $\begin{array}{l}\text { DEXi; Multi-criteria modeling; Rural tourism; service } \\
\text { quality; Tourist farm }\end{array}$ \\
\hline 139 & $\begin{array}{l}\text { Understanding the relationships of quality, value, equity, } \\
\text { satisfaction, and behavioral intentions among golf } \\
\text { travelers (2009) }\end{array}$ & $\begin{array}{l}\text { Behavioral intention; Customer loyalty; Equity; } \\
\text { Golf traveler; Satisfaction; Value }\end{array}$ \\
\hline 140 & $\begin{array}{l}\text { A visitors' evaluation index for a visit to an archaeological } \\
\text { site (2010) }\end{array}$ & $\begin{array}{l}\text { Archaeological site; Formative index; service } \\
\text { convenience; service experience; service quality }\end{array}$ \\
\hline 141 & $\begin{array}{l}\text { An exploratory inquiry into destination risk perceptions } \\
\text { and risk reduction strategies of first time vs. repeat visitors } \\
\text { to a highly volatile destination (2011) }\end{array}$ & $\begin{array}{l}\text { Motives; Repeat visitors; Risk perception; Risk } \\
\text { reduction strategies; Volatile destination }\end{array}$ \\
\hline
\end{tabular}


Table 2. Cont.

\begin{tabular}{|c|c|c|}
\hline \multirow{2}{*}{ No. } & \multicolumn{2}{|c|}{ Journals } \\
\hline & Title (Year) & Author Keywords \\
\hline \multicolumn{3}{|c|}{ Tourism Management } \\
\hline 142 & $\begin{array}{l}\text { Critical issues affecting the service quality } \\
\text { and professionalism of the tour guides in Hong Kong } \\
\text { and Macau (2011) }\end{array}$ & $\begin{array}{l}\text { Entrepreneur role; Professional habitus; } \\
\text { Professionalism; Role conflict; service quality; } \\
\text { Tour guide }\end{array}$ \\
\hline 143 & Customer satisfaction using low cost carriers (2011) & $\begin{array}{l}\text { Behavioral intentions; Customer satisfaction; Low } \\
\text { cost carriers; Perceived service quality }\end{array}$ \\
\hline 144 & $\begin{array}{l}\text { Using a modified grey relation method for improving } \\
\text { airline service quality (2011) }\end{array}$ & $\begin{array}{l}\text { Airline; Customers' needs; Grey relation; } \\
\text { Multiple-criteria decision-making (MCDM); service } \\
\text { quality; SERVQUAL }\end{array}$ \\
\hline 145 & $\begin{array}{l}\text { Comment on "Using a modified grey relation method for } \\
\text { improving airline service quality" (2012) }\end{array}$ & $\begin{array}{l}\text { Airline; Grey relation; MCDM; service quality; } \\
\text { VIKOR }\end{array}$ \\
\hline 146 & $\begin{array}{l}\text { Passengers' perceptions of airline lounges: Importance of } \\
\text { attributes that determine usage and service quality } \\
\text { measurement (2012) }\end{array}$ & $\begin{array}{l}\text { Airline lounge; Atmosphere; F\&B service; } \\
\text { service quality }\end{array}$ \\
\hline 147 & $\begin{array}{l}\text { Reply to "Comment on using a modified grey relation } \\
\text { method for improving airline service quality" (2012) }\end{array}$ & Grey relation; service quality; TOPSIS; VIKOR \\
\hline 148 & $\begin{array}{l}\text { Quality deterioration in package tours: The interplay of } \\
\text { asymmetric information and reputation (2013) }\end{array}$ & $\begin{array}{l}\text { Asymmetric information; China; Package tours; } \\
\text { Quality deterioration; Reputation }\end{array}$ \\
\hline 149 & $\begin{array}{l}\text { A novel framework for customer-driven service strategies: } \\
\text { A case study of a restaurant chain (2014) }\end{array}$ & $\begin{array}{l}\text { Customer satisfaction; Importance-performance } \\
\text { analysis; service quality; Signal-to-noise ratio; } \\
\text { The Kano model }\end{array}$ \\
\hline 150 & $\begin{array}{l}\text { Examining strategies for maximizing and utilizing brand } \\
\text { prestige in the luxury cruise industry (2014) }\end{array}$ & $\begin{array}{l}\text { Brand consciousness; Brand identification; Brand } \\
\text { prestige; Luxury cruise; Well-being perception }\end{array}$ \\
\hline 151 & $\begin{array}{l}\text { Improving importance-performance analysis: The role of } \\
\text { the zone of tolerance and competitor performance. } \\
\text { The case of Taiwan's hot spring hotels (2014) }\end{array}$ & $\begin{array}{l}\text { Benchmarking; Hot spring hotels; services quality; } \\
\text { Zone of tolerance }\end{array}$ \\
\hline 152 & $\begin{array}{l}\text { Developing similarity-based IPA under intuitionistic fuzzy } \\
\text { sets to assess leisure bikeways (2015) }\end{array}$ & $\begin{array}{l}\text { Bikeway; Importance-performance analysis; } \\
\text { Intuitionistic fuzzy set; Pattern recognition; service } \\
\text { quality; Similarity }\end{array}$ \\
\hline 153 & $\begin{array}{l}\text { Ensuring corporate travel compliance-Control vs. } \\
\text { commitment strategies (2015) }\end{array}$ & $\begin{array}{l}\text { Case study; Commitment; Control; Corporate travel; } \\
\text { service quality; service triad; Travel policy } \\
\text { compliance }\end{array}$ \\
\hline 154 & $\begin{array}{l}\text { service quality and the training of employees: } \\
\text { The mediating role of organizational commitment (2015) }\end{array}$ & $\begin{array}{l}\text { Commitment; Hotels; India; service quality; Tourist; } \\
\text { Training }\end{array}$ \\
\hline 155 & $\begin{array}{l}\text { The determinants of recommendations to use augmented } \\
\text { reality technologies: The case of a Korean theme } \\
\text { park (2015) }\end{array}$ & $\begin{array}{l}\text { Augmented reality; DeLone and McLean model; } \\
\text { Personal innovativeness; Process theory; Satisfaction; } \\
\text { Smartphone }\end{array}$ \\
\hline 156 & $\begin{array}{l}\text { Using a randomised experiment to test the causal effect of } \\
\text { service quality on visitor satisfaction and loyalty in } \\
\text { a remote national park (2015) }\end{array}$ & $\begin{array}{l}\text { Interventions; Loyalty; Protected areas; Randomised } \\
\text { experiment; Visitor satisfaction }\end{array}$ \\
\hline 157 & $\begin{array}{l}\text { A comparison of service quality attributes for stand-alone } \\
\text { and resort-based luxury hotels in Macau: 3-Dimensional } \\
\text { importance-performance analysis (2016) }\end{array}$ & $\begin{array}{l}\text { Importance-performance analysis; Luxury hotel; } \\
\text { service quality measurement scale; } \\
\text { Three-factor theory }\end{array}$ \\
\hline 158 & $\begin{array}{l}\text { Hotel attributes: Asymmetries in guest payments } \\
\text { and gains-A stated preference approach (2016) }\end{array}$ & $\begin{array}{l}\text { Discrete choice experiments; Discrete choice models; } \\
\text { Hotel choice; Willingness to accept; Willingness } \\
\text { to pay }\end{array}$ \\
\hline 159 & $\begin{array}{l}\text { Police culture, tourists and destinations: A study of } \\
\text { Uttarakhand, India (2016) }\end{array}$ & $\begin{array}{l}\text { Leader behavior; Police organization culture; service } \\
\text { quality of police; Tourists' confidence in police }\end{array}$ \\
\hline 160 & $\begin{array}{l}\text { The effects of perceived service quality on repurchase } \\
\text { intentions and subjective well-being of Chinese tourists: } \\
\text { The mediating role of relationship quality (2016) }\end{array}$ & $\begin{array}{l}\text { Customer satisfaction; Customer-company } \\
\text { identification; Repurchase intentions; service quality; } \\
\text { Subjective well-being }\end{array}$ \\
\hline 161 & $\begin{array}{l}\text { The trickle-down effect of servant leadership on frontline } \\
\text { employee service behaviors and performance: } \\
\text { A multilevel study of Chinese hotels (2016) }\end{array}$ & $\begin{array}{l}\text { Hospitality; Servant leadership; service climate; } \\
\text { service quality; service-oriented behavior; } \\
\text { Trickle-down model }\end{array}$ \\
\hline 162 & $\begin{array}{l}\text { Travel web-site design: Information task-fit, service quality } \\
\text { and purchase intention (2016) }\end{array}$ & $\begin{array}{l}\text { Empirical research; Information-task fit; Product } \\
\text { quality; Purchase intentions; Website design quality }\end{array}$ \\
\hline 163 & $\begin{array}{l}\text { An ant colony based optimization for RFID reader } \\
\text { deployment in theme parks under service level } \\
\text { consideration (2017) }\end{array}$ & $\begin{array}{l}\text { Ant colony optimization; Reader deployment; service } \\
\text { level index; Theme parks; Tracking systems }\end{array}$ \\
\hline 164 & $\begin{array}{l}\text { Assessing the effectiveness of empowerment on service } \\
\text { quality: A multi-level study of Chinese tourism } \\
\text { firms (2017) }\end{array}$ & $\begin{array}{l}\text { Cross-level; Empowerment climate; Psychological } \\
\text { empowerment; service behavior-based evaluation } \\
\text { (SBE); service quality; Tourism firms }\end{array}$ \\
\hline 165 & $\begin{array}{l}\text { Festival attributes and perceptions: A meta-analysis of } \\
\text { relationships with satisfaction and loyalty (2017) }\end{array}$ & $\begin{array}{l}\text { Attributes; Festival; Loyalty; meta-analysis; } \\
\text { Perceptions; Satisfaction }\end{array}$ \\
\hline
\end{tabular}


Table 2. Cont.

\begin{tabular}{|c|c|c|}
\hline \multirow{2}{*}{ No. } & \multicolumn{2}{|c|}{ Journals } \\
\hline & Title (Year) & Author Keywords \\
\hline \multicolumn{3}{|c|}{ Tourism Management } \\
\hline 166 & $\begin{array}{l}\text { Sources of satisfaction with luxury hotels for new, repeat, } \\
\text { and frequent travelers: A PLS impact-asymmetry } \\
\text { analysis (2017) }\end{array}$ & $\begin{array}{l}\text { Frequent travelers; Impact-asymmetry analysis; } \\
\text { Luxury hotels; Satisfaction; service quality }\end{array}$ \\
\hline 167 & $\begin{array}{l}\text { Contemplating museums??service failure: Extracting } \\
\text { the service quality dimensions of museums from negative } \\
\text { on-line reviews (2018) }\end{array}$ & $\begin{array}{l}\text { Museum management; Museum tourism; On-line } \\
\text { review; service failure; service quality; Social media; } \\
\text { TripAdvisor; Visitor experience }\end{array}$ \\
\hline 168 & $\begin{array}{l}\text { Does a happy destination bring you happiness? Evidence } \\
\text { from Swiss inbound tourism (2018) }\end{array}$ & $\begin{array}{l}\text { Happiness; Life satisfaction; Switzerland; Tourist } \\
\text { destination }\end{array}$ \\
\hline 169 & $\begin{array}{l}\text { In-flight NCCI management by combining the Kano model } \\
\text { with the service blueprint: A comparison of frequent } \\
\text { and infrequent flyers (2018) }\end{array}$ & $\begin{array}{l}\text { Airline industry; Flying frequency; In-flight NCCI; } \\
\text { Kano model; service blueprint }\end{array}$ \\
\hline 170 & $\begin{array}{l}\text { Innovation and 19th century hotel industry } \\
\text { evolution (2018) }\end{array}$ & $\begin{array}{l}\text { Hotel industry history; Niche cumulation; } \\
\text { Technological transition; Tourism history; Tourism } \\
\text { methodology }\end{array}$ \\
\hline 171 & $\begin{array}{l}\text { Is culture of origin associated with more expressions? } \\
\text { An analysis of Yelp reviews on Japanese restaurants (2018) }\end{array}$ & $\begin{array}{l}\text { Cross-cultural difference; Online restaurant review; } \\
\text { Sentiment analysis; Vocabulary range }\end{array}$ \\
\hline 172 & $\begin{array}{l}\text { Is role stress always harmful? Differentiating role overload } \\
\text { and role ambiguity in the challenge-hindrance stressors } \\
\text { framework (2018) }\end{array}$ & $\begin{array}{l}\text { Challenge-hindrance stressors; Employee } \\
\text { psychological empowerment; Hierarchical linear } \\
\text { modeling; Organizational supportive leadership } \\
\text { climate; Role ambiguity; Role overload; service } \\
\text { quality }\end{array}$ \\
\hline 173 & $\begin{array}{l}\text { Mobile social tourism shopping: A dual-stage analysis of } \\
\text { a multi-mediation model (2018) }\end{array}$ & $\begin{array}{l}\text { Artificial Neural Network analysis; Mobile social } \\
\text { tourism shopping; Multiple mediation analysis; } \\
\text { Partial Least Squares Structural Equation Modelling; } \\
\text { Stimulus-Organism-Response framework; Tourism } \\
\text { products and services }\end{array}$ \\
\hline 174 & $\begin{array}{l}\text { Past themes and future trends in medical tourism research: } \\
\text { A co-word analysis (2018) }\end{array}$ & $\begin{array}{l}\text { Bibliometric analysis; Co-word analysis; Health } \\
\text { tourism; Medical tourism; Thematic evolution }\end{array}$ \\
\hline 175 & $\begin{array}{l}\text { Predicting determinants of hotel success and development } \\
\text { using Structural Equation Modelling (SEM)-ANFIS } \\
\text { method (2018) }\end{array}$ & $\begin{array}{l}\text { Critical Success Factors (CSFs); HOT-fit Model; Hotel } \\
\text { success and development; SEM-ANFIS; TOE } \\
\text { framework; Tourism }\end{array}$ \\
\hline 176 & $\begin{array}{l}\text { Quality assessment of airline baggage handling systems } \\
\text { using SERVQUAL and BWM (2018) }\end{array}$ & $\begin{array}{l}\text { Baggage handling; Best worst method; BWM; } \\
\text { Quality; SERVQUAL }\end{array}$ \\
\hline 177 & $\begin{array}{l}\text { Cooperation and competition between online travel } \\
\text { agencies and hotels (2019) }\end{array}$ & $\begin{array}{l}\text { competition; Cooperation; hotel; } \mathrm{O} 2 \mathrm{O} \text { commerce; } \\
\text { Online travel agency }\end{array}$ \\
\hline 178 & $\begin{array}{l}\text { What do hotel customers complain about? Text analysis } \\
\text { using structural topic model (2019) }\end{array}$ & $\begin{array}{l}\text { Customer dissatisfaction; Online hotel reviews; } \\
\text { Structural topic model; Text mining; Trip advisor }\end{array}$ \\
\hline
\end{tabular}

\section{Bibliometric and Network Analysis}

We briefly conducted a two-part data analysis comprising bibliometric analysis and network analysis (which included citation analysis and keyword network analysis) using NetMiner 4.0, a network analysis software that enables the analysis of not only network data but also unstructured text data. Using the bibliometric approach, we analyzed the frequency of titles and keywords in paper texts and abstracts to reveal a given paper's importance and then identify critical issues and trends. Unlike bibliometric analysis, the network analysis was performed to investigate core research issues and topics by constructing networks based on the co-citation of papers and co-occurrence of keywords.

\subsection{Bibliometric Analysis}

To perform the bibliometric analysis, we used additional data, such as the frequency of titles, authors, journals, and keywords. NetMiner was used to extract frequent words in the titles and keywords in selected papers and analyze the constructed networks.

The results of the bibliometric analysis are summarized in Table 3. Initially, 539 words were extracted from titles and 1595 words from abstracts. We determined the importance of these words based on their frequency of appearance. Words identified as important, shown in Table 3, include "service quality," "service," "hotel," "model," and "satisfaction." These results indicate that the included tourism service quality studies are mainly focused on the hotel industry and service satisfaction. 
Table 3. The most frequently used words.

\begin{tabular}{|c|c|c|c|c|c|}
\hline \multirow[t]{2}{*}{ No. } & \multicolumn{2}{|c|}{$\begin{array}{l}\text { The Most Frequently Used } \\
\text { Words in Titles }\end{array}$} & \multirow[t]{2}{*}{ No. } & \multicolumn{2}{|c|}{$\begin{array}{l}\text { The Most Frequently Used } \\
\text { Words in Abstracts }\end{array}$} \\
\hline & Word & Frequency & & Word & Frequency \\
\hline 1 & service quality & 42 & 1 & service quality & 259 \\
\hline & customer & & 2 & study & 239 \\
\hline 2 & service & 21 & 3 & service & 154 \\
\hline 4 & effect & 20 & 4 & customer & 145 \\
\hline 5 & role & 19 & 5 & result & 132 \\
\hline 6 & restaurant & 18 & 6 & hotel & 116 \\
\hline 7 & hotel & 16 & 7 & research & 108 \\
\hline 8 & perception & 11 & 8 & relationship & 106 \\
\hline & model & & 9 & satisfaction & 97 \\
\hline 9 & satisfaction & 9 & 10 & model & 95 \\
\hline
\end{tabular}

\subsection{Network-Based Analysis: Citation Network Analysis}

Citation analysis has become more widespread because of its ability to objectively identify influential papers in a given area [16-18]. According to Ding and Cronin [12], citation analysis is primarily focused on identifying the popularity and significance of a published paper by counting how frequently that paper is cited by other papers [19].

To continue with our systematic literature review, we constructed a local citation network for analysis that determined how many times a published paper had been cited by other published papers within a local network comprised of the 178 initially selected papers. Then, we examined three structural characteristics of the citation network: density, distance, and clustering. Density measures the proportion of actual connections in a network relative to the number of connections possible, thereby revealing the size of the network. A large network is generally sparser. Distance refers to the average number of steps along the shortest paths for all pairs of published papers, indicating the degree of information efficiency on a network. Finally, the clustering coefficient reflects the degree of connection between a published paper and neighboring papers. This coefficient is based on the ratio of the number of existing links to the number of possible links among neighboring published papers. As shown in Table 4, the local citation network of published papers on service quality in tourism is relatively sparse and highly clustered.

Table 4. The characteristics of network structure.

\begin{tabular}{ccccc}
\hline & Papers & Density & Distance & Clustering Coefficient \\
\hline All & 178 & 0.01 & 1.397 & 1.614 \\
\hline
\end{tabular}

We used the PageRank measure to further identify core papers within the subset of 178 papers. PageRank, introduced by Brin and Page [20], was created to prioritize web pages in the Google search engine [19]. PageRank can be used to measure "prestige," an important indicator of webpage quality, by revealing the number of times a paper is cited by other highly cited papers. The PageRank of paper $\mathrm{A}$ (denoted by $\mathrm{PR}(\mathrm{A})$ ) in a network constructed with $\mathrm{N}$ papers can be computed as follows:

$$
\frac{(1-d)}{N}+d\left(\frac{P R\left(T_{1}\right)}{C\left(T_{1}\right)}+\cdots \cdots+\frac{P R\left(T_{n}\right)}{C\left(T_{n}\right)}\right)
$$

where paper $T_{i}$ has citations $C\left(T_{i}\right)$ and $d$ is a damping factor representing the fraction of random walks that continue to propagate along the citations. In our study, the parameter $d$ was chosen to be 0.85 based on Brin and Page [20].

Table 5 indicates the top 10 papers as analyzed by PageRank. These papers can be regarded as core papers in the field of tourism service quality and mainly address the importance of service quality 
for customer satisfaction and its relationship with customer behavior. For example, Kim et al. [21] investigated the impact of tourism service quality on customer behavior according to customer satisfaction. Chen et al. [22] examined the determinants of customer participation in service encounters and their impact on customer loyalty. Ladhari et al. [23] analyzed the importance of service quality as a factor in dining satisfaction with regard to restaurant services. Ha and Jang [24] also studied the relationship between service quality and customer satisfaction regarding its effect on loyalty in Korean restaurants. Hutchinson et al. [25] attempted to clarify the impacts of service quality and customer satisfaction on customer behavioral intention. Additionally, some of the 10 papers dealt with service quality evaluation. For instance, Hsieh et al. [26] devised a service quality evaluation framework for hot spring hotels, and Martínez Caro and Martínez García [27] researched a comprehensive model to measure service quality in tourism-related sectors.

Table 5. Top 10 papers according to the PageRank algorithm.

\begin{tabular}{|c|c|c|}
\hline \multicolumn{3}{|c|}{ Influential Papers } \\
\hline No. & Title & PageRank \\
\hline 1 & $\begin{array}{l}\text { [21] } \\
\begin{array}{l}\text { Influence of institutional DINESERV on customer satisfaction, return intention, } \\
\text { and word-of-mouth }\end{array}\end{array}$ & 0.018510 \\
\hline 2 & $\begin{array}{l}{[22]} \\
\text { Antecedents of mandatory customer participation in service encounters: } \\
\text { An empirical study }\end{array}$ & 0.007353 \\
\hline 3 & $\begin{array}{c}\text { [28] } \\
\text { Construction and validation of the customer participation scale }\end{array}$ & \\
\hline 4 & $\begin{array}{c}{[23]} \\
\text { Determinants of dining satisfaction and post-dining behavioral intentions }\end{array}$ & 0.006856 \\
\hline 5 & $\begin{array}{l}{[24]} \\
\text { Effects of service quality and food quality: The moderating role of atmospherics in } \\
\text { an ethnic restaurant segment }\end{array}$ & 0.005837 \\
\hline 6 & $\begin{array}{l}\text { Customer value in the hotel industry: What managers believe they deliver and what } \\
\text { customer experience }\end{array}$ & 0.004211 \\
\hline 7 & $\begin{array}{c}{[26]} \\
\text { A service quality measurement architecture for hot spring hotels in Taiwan }\end{array}$ & 0.003915 \\
\hline 8 & $\begin{array}{l}{[30]} \\
\text { Using a modified grey relation method for improving airline service quality }\end{array}$ & 0.003915 \\
\hline 9 & $\begin{array}{l}\text { U25] } \\
\begin{array}{c}\text { Understanding the relationships of quality, value, equity, satisfaction, and behavioral } \\
\text { intentions among golf travelers }\end{array}\end{array}$ & 0.003845 \\
\hline 10 & $\begin{array}{c}\text { Developing a multidimensional and hierarchical service quality model for the travel } \\
\text { agency industry }\end{array}$ & 0.003835 \\
\hline
\end{tabular}

Note: This selection was taken from the 178 papers that were initially selected.

Likewise, we further conducted a co-citation analysis to identify prevalent topics through the co-occurrence of two given papers in other papers [31]. The papers comprising the co-citation network were classified into several clusters, in which the links between the articles in the given cluster were greater than those of other clusters [31-33]. In our study, clusters' index $Q$ was calculated by using the algorism [32] as follows:

$$
\mathrm{Q}=\frac{1}{2 m} \sum_{v w}\left[A_{v w}-\frac{k_{v} k_{w}}{2 m}\right] \delta\left(c_{v}, c_{w}\right)
$$


where $A_{v w}$ represents the weight of the edge between nodes $v$ and $w, k_{v}$ expresses the sum of the weights of the edges attached to node $v\left(k_{v}=\sum_{w} A_{v w}\right), c_{v}$ is the community to which node $v$ is assigned, $\delta(i, j)$ is equal to 1 if $i=j$ and 0 otherwise, and $m=\frac{1}{2} \sum_{v w} A_{v w}$.

Table 6 shows the local citation network's division into seven topical issue clusters. To identify the core topical issues for each cluster, we determined the lead papers in each cluster by using the PageRank algorithm. Then, a general description for each cluster's topic was ascertained using these lead papers.

Table 6. The lead papers using the PageRank algorithm for each cluster.

\begin{tabular}{|c|c|c|}
\hline Clusters & & Top Three Lead Papers According to the PageRank Algorithm \\
\hline \multirow{3}{*}{1} & [34] & $\begin{array}{l}\text { A systematic approach to scale development in tourist shopping satisfaction: Linking } \\
\text { destination attributes and shopping experience }\end{array}$ \\
\hline & [35] & Enhancing service loyalty: The roles of delight, satisfaction, and service quality \\
\hline & [36] & $\begin{array}{l}\text { Festival attributes and perceptions: A meta-analysis of relationships with satisfaction } \\
\text { and loyalty }\end{array}$ \\
\hline \multirow{3}{*}{2} & [37] & $\begin{array}{l}\text { An investigation of green hotel customers' decision formation: Developing } \\
\text { an extended model of the theory of planned behavior }\end{array}$ \\
\hline & [13] & Less is more: A new insight for measuring service quality of green hotels \\
\hline & [38] & $\begin{array}{l}\text { The impact of green practices in coastal tourism: An empirical investigation on an eco- } \\
\text { labeled beach club }\end{array}$ \\
\hline \multirow{3}{*}{3} & [29] & $\begin{array}{l}\text { Customer value in the hotel industry: What managers believe they deliver and what } \\
\text { customer experience }\end{array}$ \\
\hline & [25] & $\begin{array}{l}\text { Understanding the relationships of quality, value, equity, satisfaction, and behavioral } \\
\text { intentions among golf travelers }\end{array}$ \\
\hline & [39] & $\begin{array}{l}\text { Pay for performance and work attitudes: The mediating role of employee-organization } \\
\text { service value congruence }\end{array}$ \\
\hline \multirow{3}{*}{4} & [40] & $\begin{array}{l}\text { Influence of the quality of food, service, and physical environment on customer } \\
\text { satisfaction and behavioral intention in quick-casual restaurants: Moderating role of } \\
\text { perceived price }\end{array}$ \\
\hline & [41] & Restaurant consumers repeat patronage: A service quality concern \\
\hline & [42] & Foreign travelers' satisfaction with traditional Korean restaurants \\
\hline \multirow{3}{*}{5} & [43] & $\begin{array}{l}\text { Relationships among experiential marketing, experiential value, and customer } \\
\text { satisfaction }\end{array}$ \\
\hline & [44] & $\begin{array}{l}\text { The role and effect of job satisfaction and empowerment on customers' perception of } \\
\text { service quality: A study in the restaurant industry }\end{array}$ \\
\hline & [45] & $\begin{array}{l}\text { An exploratory examination of supervisor undermining, employee involvement } \\
\text { climate, and the effects on customer perceptions of service quality in quick-service } \\
\text { restaurants }\end{array}$ \\
\hline \multirow{3}{*}{6} & {$[26]$} & A service quality measurement architecture for hot spring hotels in Taiwan \\
\hline & [27] & $\begin{array}{l}\text { Developing a multidimensional and hierarchical service quality model for the travel } \\
\text { agency industry }\end{array}$ \\
\hline & [46] & An examination of electronic video clips in the context of hotel Websites \\
\hline \multirow{3}{*}{7} & [21] & $\begin{array}{l}\text { Influence of institutional DINESERV on customer satisfaction, return intention, } \\
\text { and word-of-mouth }\end{array}$ \\
\hline & [22] & Determinants of dining satisfaction and post-dining behavioral intentions \\
\hline & [24] & $\begin{array}{l}\text { Effects of service quality and food quality: The moderating role of atmospherics in } \\
\text { an ethnic restaurant segment }\end{array}$ \\
\hline
\end{tabular}


Cluster 1 corresponded to tourist satisfaction from various perspectives. For example, Wong and Wan [34] explored tourists' shopping satisfaction and examined its dimensionality. Ahrholdt et al. [35] also investigated tourists' satisfaction and loyalty according to prior experience. Tanford and Jung [36] evaluated the factors contributing to tourists' festival satisfaction.

Cluster 2 related to sustainable issues in tourism. Han and Kim [37] examined tourists' intention to revisit a green hotel. Lee and Cheng [13] investigated tourists' decision-making process in terms of staying at a green hotel. Most recently, Merli et al. [38] addressed tourists' perception of green practices and the impact of these practices on their satisfaction and loyalty.

Cluster 3 corresponded to the value customers place on tourism service quality. For example, Nasution and Mavondo [29] viewed customer value from two perspectives: that of the service provider, and that of the customer. Hutchinson et al. [25] addressed the effect of service evaluations, including value, on customer intentions. Chiang and Birtch [39] researched the effect of service value congruence between the employee and organization on pay-for-performance and work attitudes.

Cluster 4 mainly focused on service quality in restaurants and its impact on customer satisfaction. For example, Ryu and Han [40] examined the effect of restaurant food and service quality on customer intention. Barber et al. [41] aimed to determine the relationship between restaurant cleanliness and customers' repeated patronage. Nam and Lee [42] investigated the factors related to foreign tourists' satisfaction with traditional Korean restaurants.

Cluster 5 generally included customer perceptions regarding service quality. For example, Yuan and $\mathrm{Wu}$ [43] focused on the emotional and functional values made by service quality. Gazzoli et al. [44] discussed the relationship of empowerment and job satisfaction to customers' perception of service quality. Mathe and Slevitch [45] explored the factors impacting customers' perception of service quality.

Cluster 6 contained various assessments of service quality to identify service quality's effect on customer satisfaction. For example, Hsieh et al. [26] analyzed customers' expectations for hotel service quality according to its five dimensions. Likewise, Martínez Caro and Martínez García [27] developed a comprehensive model to measure service quality in tourism. Kim and Mattila [46] also studied customer evaluations regarding hotel service through six distinct dimensions.

Lastly, Cluster 7 corresponded to how service quality affects customer behavior. For instance, Kim et al. [21] analyzed the effect of service quality on customers' intention to return and word-of-mouth endorsement. Ladhari et al. [23] determined dining satisfaction factors in terms of restaurant service and its relationship to customer behaviors such as loyalty and willingness to pay more. Similarly, Ha and Jang [24] examined the relationship between perceived quality by customers and restaurant loyalty.

To understand the evolution of tourism service quality research over time, we also conducted a dynamic co-citation analysis of analyzed articles to indicate the evolution/development of each cluster over time. (Table 7)

Table 7. Research focus of each cluster.

\begin{tabular}{cc}
\hline Cluster & Main Topic \\
\hline 1 & Tourist satisfaction \\
2 & Sustainable issues in tourism \\
3 & Value of service quality for customers \\
4 & Restaurant service quality \\
5 & Customer perception of tourism service quality \\
6 & Tourism service quality assessment \\
7 & Customer behavior \\
\hline
\end{tabular}

As seen in Table 8, earlier publications corresponded to Clusters 1, 3, 5, 6, and 7. Significantly, development of the topics corresponding to Clusters 1,5, and 7 has diminished while that of Clusters 2 and 4 has continued to grow. Furthermore, the number of research papers focusing on the topics in Clusters 2 and 3 has steadily increased. 
Table 8. The number of published papers in each cluster (2008-2019).

\begin{tabular}{|c|c|c|c|c|c|c|c|}
\hline Year & Cluster 1 & Cluster 2 & Cluster 3 & Cluster 4 & Cluster 5 & Cluster 6 & Cluster 7 \\
\hline 2008 & 1 & & 1 & & 1 & 2 & 1 \\
\hline 2009 & & & 2 & & & & 1 \\
\hline 2010 & & 1 & 1 & 1 & 1 & 2 & 3 \\
\hline 2011 & & & 2 & 3 & & 2 & 1 \\
\hline 2012 & & & & & & & 3 \\
\hline 2013 & 1 & 1 & 1 & 1 & 1 & & 2 \\
\hline 2014 & & & & 1 & 1 & 1 & 3 \\
\hline 2015 & & & 2 & 1 & & & 3 \\
\hline 2016 & & & 1 & & & 2 & 1 \\
\hline 2017 & 2 & & 1 & 3 & 1 & 1 & 2 \\
\hline 2018 & & 1 & 1 & 1 & & 2 & 1 \\
\hline 2019 & & 1 & & 1 & & & \\
\hline Total & 4 & 4 & 12 & 12 & 5 & 12 & 21 \\
\hline
\end{tabular}

\subsection{Network-Based Analysis: Keyword Network Analysis}

After performing the citation network analysis, we executed a keyword network analysis based on 608 keywords extracted from 178 papers.

To conduct the keyword network analysis, we followed the process summarized in Table 9. First, we (1) constructed a keyword network using keywords extracted from premiere international tourism-focused academic journals (namely, The Annals of Tourism Research, International Journal of Hospitality Management, Journal of Hospitality and Tourism Research, Journal of Travel Research, and Tourism Management). We then (2) investigated the issues and topics related to service in the tourism sector utilizing network analysis. Finally, we (3) observed the changes in the issues and topics that occurred from 2008 to 2019.

Table 9. Keyword network analysis process.

\begin{tabular}{|c|c|c|}
\hline \multicolumn{3}{|c|}{ Analysis Process } \\
\hline \multirow{3}{*}{ Network construction } & \multirow{3}{*}{ Keyword network construction } & Refinement of search keywords \\
\hline & & $\begin{array}{l}\text { Construction of keyword network based on the frequency of } \\
\text { keyword co-occurrence }\end{array}$ \\
\hline & & $\begin{array}{l}\text { Construction of commonly addressed keyword network based } \\
\text { on component analysis }\end{array}$ \\
\hline \multirow[t]{2}{*}{ Network analysis } & \multirow[t]{2}{*}{ Keyword network analysis } & Cluster analysis \\
\hline & & Network centrality analysis by year \\
\hline
\end{tabular}

More specifically, in the afore-mentioned step 1, we used 608 keywords to form a network for tourism service quality. Before constructing a keyword network, we refined the keywords extracted from 178 papers by standardizing keywords that had the same fundamental meaning. The rules used to refine the keywords [11] are as follows:

- Standardization into a singular form

- Removing redundant keywords

- Removing hyphens

- Avoidance of abbreviations

- Unification of synonyms

- Separation of multiple terms in a single keyword

Thus, standardization resulted in 604 relevant keywords from the original list of 629 keywords. To construct the network consisting of the most important, commonly referenced keywords, we then 
performed component analysis using NetMiner. A component in a network indicates a group of nodes (papers) that are all connected to each other, representing commonly addressed issues and topics in the network.

\subsubsection{Keyword Network Analysis: Network Centrality Analysis}

After performing the component analysis, we were able to observe differences in the classification of the top 10 keywords across three different measures: degree, betweenness, and closeness centrality. As shown in Table 10, the differences in the top-ranked keywords according to the three measures implies that research on tourism service focuses on both broad and specific issues. The top 10 keywords according to the centrality measures are important in terms of their structural positions in the keyword network.

Table 10. Top 10 keywords across measures.

\begin{tabular}{cccc}
\hline Rank & Degree Centrality & Betweenness Centrality & Closeness Centrality \\
\hline 1 & satisfaction & satisfaction & satisfaction \\
2 & customer satisfaction & customer satisfaction & customer satisfaction \\
3 & value & China & value \\
4 & behavioral intention & behavioral intention & behavioral intention \\
5 & online review & emotion & online review \\
6 & equity & online review & equity \\
7 & loyalty & service failure & loyalty \\
8 & emotion & equity & emotion \\
9 & perceived value & electronic word of mouth & perceived value \\
10 & customer loyalty & perceived quality & customer loyalty \\
\hline
\end{tabular}

The top 10 keywords according to the degree of centrality are "satisfaction," "customer satisfaction," "value," "behavioral intention," "online review," "equity," "loyalty," "emotion," "perceived value," and "customer satisfaction," and these words represent important keywords in terms of their structural position in the keywords network. These keywords have many connections with other keywords, which indicates that they represent major research issues in the field of tourism service quality.

The top 10 keywords according to betweenness centrality_"satisfaction," "customer satisfaction," "China," "behavioral intention," "emotion," "online review," "service failure," "equity," "electronic word-of-mouth," and "perceived quality" - play an important role in bridging separated groups of research themes. In other words, these keywords lie between two distinctive research themes.

Finally, the top 10 keywords in terms of closeness centrality are "satisfaction," "customer satisfaction", "value," "behavioral intention," "online review," "equity," "loyalty," "emotion," "perceived value," and "customer loyalty." These words were used with nearly all other keywords and themes in the network because a keyword with high closeness centrality is located in the center of the keyword network.

\subsubsection{Keyword Network Analysis: Changes in Important Keywords Over Time}

To address the changes in the important keywords over time, we compared the important keywords from the first nine years (2008-2016) with those from the three most recent years (2017-2019). We then compared high-ranked keywords across the three network measurements (see Table 11). It is important to note that the connections between keywords have accumulated over years, making it inherently difficult to investigate the evolution of keyword networks. In other words, although the keyword network constructed for a certain period of time offers information about the associations among the keywords for the published papers in that specific period, it is possible to exclude significant information regarding keyword associations in other periods [11]. The associations among keywords across different time periods do affect one another; thus, they are correlated [11]. This is a common issue when investigating the evolution of citation, author, and keyword networks. Comparing the keyword 
network corresponding to data obtained from much earlier studies with that from more recent studies can mitigate the potential loss of information concerning recent changes in impactful keywords [11].

Table 11. Comparison of the top five keywords across three network measurements.

\begin{tabular}{|c|c|c|c|c|c|c|}
\hline \multirow{3}{*}{$\begin{array}{c}\text { Rank } \\
1\end{array}$} & $2008-2016$ & $2017-2019$ & 2008-2016 & $2017-2019$ & $2008-2016$ & $2017-2019$ \\
\hline & \multicolumn{2}{|c|}{ Degree Centrality } & \multicolumn{2}{|c|}{ Closeness Centrality } & \multicolumn{2}{|c|}{ Betweenness Centrality } \\
\hline & service quality & service quality & service quality & service quality & service quality & service quality \\
\hline 2 & satisfaction & hospitality industry & satisfaction & trip advisor & satisfaction & customer satisfaction \\
\hline 3 & customer satisfaction & trip advisor & customer satisfaction & loyalty & China & trip advisor \\
\hline 4 & loyalty & loyalty & loyalty & hospitality industry & customer satisfaction & hospitality industry \\
\hline 5 & equity & satisfaction & equity & satisfaction & emotion & loyalty \\
\hline
\end{tabular}

Our comparison reveals some notable findings. "Service quality," "satisfaction," "customer satisfaction," and "loyalty" were the most important keywords for all three measures for both clustered time periods. Significantly, customer satisfaction-related keywords (such as "customer satisfaction," "satisfaction," and "loyalty") have received growing attention over the nine-year time period. Additionally, tourism management-related keywords (such as "trip advisor" and "hospitality industry") have become substantially more prevalent over the years.

\section{Conclusions}

Service quality has been established as an important economic impetus of tourism. To explore how this factor has been represented in past tourism literature, we conducted a systematic literature review combining bibliometric, citation network, and keyword network analysis. Furthermore, our study attempted to identify how important keywords have changed over time to capture the emerging critical issues and topical trends in tourism service research.

This study has significant implications for both theory and practice in several ways. First, due to the existence of diverse tourism sub-sectors across business settings, previous reviews on service quality have mainly focused on service quality with regard to specific themes pre-identified by the author. In contrast, the present study represents a more comprehensive literature review on service quality in tourism research by applying a systematic approach.

Second, we identified that the keyword network of service quality in tourism is relatively small, characterized by low density, short distance, and fewer clusters. It is possible that new issues and concepts related to service quality in tourism have not emerged as rapidly [11] because relatively large networks with less connectivity to neighboring research areas do not easily facilitate the creation of new issues and concepts.

Third, based on the citation analysis of research on tourism service quality, we identified critical issues most commonly discussed by influential papers. To conduct content analysis, we rigorously identified papers as "more important" by using not only the frequency of citations but also the degree of prestige, established through the PageRank measure. This is a significant methodological development, as previous studies have only used the frequency of citation to assess importance. The critical issues identified in this study concern how service quality affects tourists' behavior and service quality evaluation. Current research on service quality in tourism is still focused on the impact of service quality on customer satisfaction and behavior.

Fourth, this study extends and supports the previous systematic literature review on service quality provided by Lai et al. [15]. Their review suggested 17 research topics that comprise two main research streams: (1) service quality scares, and (2) the consequences of service quality. According to the co-citation analysis presented in this article, research on tourism service quality can actually be classified into seven topic clusters: (1) tourist satisfaction, (2) sustainable issues in tourism, (3) value of service quality for customers, (4) restaurant service quality, (5) customer perception of tourism service quality, (6) tourism service quality evaluation, and (7) service quality's influence on customer behavior.

Fifth, because important issues and topics were selected from a local citation network comprising 136 cited papers within the total 178 papers used in our study, it was difficult to identify comprehensive 
issues and topics due to the exclusion of the remaining 42 papers from the network configuration. Therefore, we implemented a keyword network analysis to complement the drawbacks of citation and co-citation analysis. The keyword network analysis revealed differences in the classification of important keywords across centrality measures. Our results reveal some notable findings. "Satisfaction" and "customer satisfaction" are represented as major research issues in the area of service quality in tourism. Also, "satisfaction" and "customer satisfaction" have not only a high degree of centrality but also high betweenness and closeness centrality. These results indicate that these are important service quality issues, and the existing literature has focused on them. Thus, service quality issues related to "satisfaction" and/or "customer satisfaction" might be a good starting point for researching the overall topic of service quality in tourism.

Finally, to identify changes in and the development of topics over time, we performed a dynamic co-citation analysis. These results showed that "sustainable issues in tourism" and "restaurant service quality" have gained researchers' attention in recent years, whereas the focus on topics such as "tourist satisfaction," "customer perception of tourism service quality," and "service quality's influence on customer behavior" has decreased. This current trend suggests an increasing interest in investigating service quality regarding sustainability (green hotel and green practice) and restaurants. Thus, this paper suggests two research areas that deserve further investigation and research: (1) service quality and sustainability, and (2) service quality and restaurants.

Customer satisfaction-related keywords (such as "customer satisfaction," "satisfaction," and "loyalty") have received growing attention over the nine-year period used in the study, while the importance of tourism management-related keywords (such as "trip advisor" and "hospitality industry") has also substantially increased during the same period. The results of this study reveal the most frequently used words in research titles and abstracts in the field of tourism service quality. After using "service quality" as the main keyword to extract significant papers, "service quality" emerged as the most frequently used word in titles and abstracts. Moreover, "hotel" and "restaurant" are also included in the list of popular keywords. This indicates that tourism service quality is largely associated with hotels and restaurants compared to other tourist destinations.

Although our study has interesting implications for service quality tourism research, this study is not free of limitations. Despite providing a comprehensive systematic review of this area, the manual search method used to retrieve the articles may have excluded or overlooked other relevant articles. As the papers were retrieved only from SCOPUS, any related articles that could not be listed in one of these databases were excluded. In addition, we choose our keywords according to our research topic. The keywords used may not be exhaustive. Expanding the keywords to reflect "service quality" could result in a more exhaustive review of the field.

Author Contributions: J.P. and E.J. conceived of and designed the methodology; J.P. conducted the literature review and collected data; E.J. performed the analysis; J.P. and E.J. contributed to the interpretation of results and discussion; J.P. and E.J. wrote the paper.

Funding: This research received no external funding.

Acknowledgments: This research is (partially) supported by the BK21PLUS Research Fund for Korea University Business School.

Conflicts of Interest: The authors declare no conflict of interest.

\section{References}

1. UNWTO World Tourism Organization. Available online: http://mkt.unwto.org (accessed on 21 May 2019).

2. Li, K.X.; Jin, M.; Shi, W. Tourism as an important impetus to promoting economic growth: A critical review. Tour. Manag. Perspect. 2018, 26, 135-142. [CrossRef]

3. Brida, J.G.; Pereyra, J.S.; Devesa, M.J.S. Evaluating the contribution of tourism to economic growth. Anatolia 2008, 19, 351-357. [CrossRef]

4. Khan, H.; Seng, C.F.; Cheong, W.K. Tourism multiplier effects on Singapore. Ann. Tour. Res. 1990, 17, 408-418. [CrossRef] 
5. Chen, C.-F. Applying the stochastic frontier approach to measure hotel managerial efficiency in Taiwan. Tour. Manag. 2007, 28, 696-702. [CrossRef]

6. Dwyer, L.; Forsyth, P.; Spurr, R. Contrasting the uses of TSAs and CGE models: Measuring tourism yield and productivity. Tour. Econ. 2007, 13, 537-551. [CrossRef]

7. Goncalves, O. Efficiency and productivity of French ski resorts. Tour. Manag. 2013, 36, 650-657. [CrossRef]

8. Meesala, A.; Paul, J. Service quality, consumer satisfaction and loyalty in hospitals: Thinking for the future. J. Retail. Consum. Serv. 2018, 40, 261-269. [CrossRef]

9. Rauch, D.A.; Collins, M.D.; Nale, R.D.; Barr, P.B. Measuring service quality in mid-scale hotels. Int. J. Contemp. Hosp. Manag. 2015, 27, 87-106. [CrossRef]

10. Transfield, D.; Denyer, D.; Smart, P. Towards a methodology for developing evidence-informed management knowledge by means of systematic review. Br. J. Manag. 2003, 14, 207-222. [CrossRef]

11. Choi, J.H.; Yi, S.; Lee, K.C. Analysis of keyword networks in MIS research and implication for predicting knowledge evolution. Inf. Manag. 2011, 48, 371-381. [CrossRef]

12. Ding, Y.; Cronin, B. Popular and/or prestigious? Measures of scholarly esteem. Inf. Process. Manag. 2011, 47, 80-96. [CrossRef]

13. Lee, W.-H.; Cheng, C.-C. Less is more: A new insight for measuring service quality of green hotels. Int. J. Hosp. Manag. 2018, 68, 32-40. [CrossRef]

14. Hjørland, B. Citation analysis: A social and dynamic approach to knowledge organization. Inf. Process. Manag. 2013, 49, 1313-1325. [CrossRef]

15. Lai, I.K.W.; Hitchcock, M.; Yang, T.; Lu, T.-W. Literature review on service quality in hospitality and tourism (1984-2014): Future directions and trends. Int. J. Contemp. Hosp. Manag. 2017, 30, 114-159. [CrossRef]

16. Culnan, M.J.; O’Reilly, C.A., III; Chatman, J.A. Intellectual structure of research in organizational behavior, 1972-1984: A cocitation analysis. J. Assoc. Inf. Sci. Technol. 1990, 41, 453-458. [CrossRef]

17. Culnan, M.J. Mapping the intellectual structure of MIS, 1980-1985: A co-citation analysis. Manag. Inf. Syst. Q. 1987, 11, 341-353. [CrossRef]

18. Gundolf, K.; Filser, M. Management research and religion: A citation analysis. J. Bus. Ethics 2013, 112, $177-185$. [CrossRef]

19. Fahimnia, B.; Sarks, J.; Davarzani, H. Green supply chain management: A review and bibliometric analysis. Int. J. Prod. Econ. 2015, 162, 101-114. [CrossRef]

20. Brin, S.; Page, L. The anatomy of a large-scale hypertextual Web search engine. Comput. Netw. ISDN Syst. 1998, 30, 107-117. [CrossRef]

21. Kim, W.G.; Ng, C.Y.N.; Kim, Y.-S. Influence of institutional DINESERV on customer satisfaction, return intention, and word-of-mouth. Int. J. Hosp. Manag. 2009, 28, 10-17. [CrossRef]

22. Chen, S.C.; Raab, C.; Tanford, S. Antecedents of mandatory customer participation in service encounters: An empirical study. Int. J. Hosp. Manag. 2015, 46, 65-75. [CrossRef]

23. Ladhari, R.; Brun, I.; Morales, M. Determinants of dining satisfaction and post-dining behavioral intentions. Int. J. Hosp. Manag. 2008, 27, 563-573. [CrossRef]

24. Ha, J.; Jang, S. Effects of service quality and food quality: The moderating role of atmospherics in an ethnic restaurant segment. Int. J. Hosp. Manag. 2010, 29, 520-529. [CrossRef]

25. Hutchinson, J.; Lai, F.; Wang, Y. Understanding the relationships of quality, value, equity, satisfaction, and behavioral intentions among golf travelers. Tour. Manag. 2009, 30, 298-308. [CrossRef]

26. Hsieh, L.-F.; Lin, L.-H.; Lin, Y.-Y. A service quality measurement architecture for hot spring hotels in Taiwan. Tour. Manag. 2008, 29, 429-438. [CrossRef]

27. Caro, L.M.; García, J.A.M. Developing a multidimensional and hierarchical service quality model for the travel agency industry. Tour. Manag. 2008, 29, 706-720. [CrossRef]

28. Chen, S.C.; Raab, C. Construction and validation of the customer participation scale. J. Hosp. Tour. Res. 2017, 41, 131-153. [CrossRef]

29. Nasution, H.N.; Mavondo, F.T. Customer value in the hotel industry: What managers believe they deliver and what customer experience. Int. J. Hosp. Manag. 2008, 27, 204-213. [CrossRef]

30. Liou, J.J.H.; Hsu, C.-C.; Yeh, W.-C.; Lin, R.-H. Using a modified grey relation method for improving airline service quality. Tour. Manag. 2011, 32, 1381-1388. [CrossRef]

31. Leydesdorff, L. Bibliometrics/Citation Networks. In Encyclopedia of Social Networks; Barnett, G.A., Ed.; SAGE Publications, Inc.: Thousand Oaks, CA, USA, 2011; pp. 73-94. 
32. Clauset, A.; Newman, M.E.J.; Moore, C. Finding community structure in very large networks. Phys. Rev. E 2004, 70, 066111. [CrossRef]

33. Radicchi, F.; Castellano, C.; Cecconi, F.; Loreto, V.; Parisi, D. Defining and identifying communities in networks. Proc. Natl. Acad. Sci. USA 2004, 101, 2658-2663. [CrossRef] [PubMed]

34. Wong, I.A.; Wan, Y.K.P. A systematic approach to scale development in tourist shopping satisfaction: Linking destination attributes and shopping experience. J. Travel Res. 2013, 52, 29-41. [CrossRef]

35. Ahrholdt, D.C.; Gudergan, S.P.; Ringle, C.M. Enhancing service loyalty: The roles of delight, satisfaction, and service quality. J. Travel Res. 2017, 56, 436-450. [CrossRef]

36. Tanford, S.; Jung, S. Festival attributes and perceptions: A meta-analysis of relationships with satisfaction and loyalty. Tour. Manag. 2017, 61, 209-220. [CrossRef]

37. Han, H.; Kim, Y. An investigation of green hotel customers' decision formation: Developing an extended model of the theory of planned behavior. Int. J. Hosp. Manag. 2010, 29, 659-668. [CrossRef]

38. Merli, R.; Preziosi, M.; Acampora, A.; Lucchetti, M.C.; Ali, F. The impact of green practices in coastal tourism: An empirical investigation on an eco-labelled beach club. Int. J. Hosp. Manag. 2019, 77, 471-482. [CrossRef]

39. Chiang, F.F.T.; Birtch, T.A. Pay for performance and work attitudes: The mediating role of employee-organization service value congruence. Int. J. Hosp. Manag. 2010, 29, 632-640. [CrossRef]

40. Ryu, K.; Han, H. Influence of the quality of food, service, and physical environment on customer satisfaction and behavioral intention in quick-casual restaurants: Moderating role of perceived price. J. Hosp. Tour. Res. 2010, 34, 310-329. [CrossRef]

41. Barber, N.; Goodman, R.J.; Goh, B.K. Restaurant consumers repeat patronage: A service quality concern. Int. J. Hosp. Manag. 2011, 30, 329-336. [CrossRef]

42. Nam, J.-H.; Lee, T.J. Foreign travelers' satisfaction with traditional Korean restaurants. Int. J. Hosp. Manag. 2011, 30, 982-989. [CrossRef]

43. Yuan, Y.-H.; Wu, C. Relationships among experiential marketing, experiential value, and customer satisfaction. J. Hosp. Tour. Res. 2008, 32, 387-410. [CrossRef]

44. Gazzoli, G.; Hancer, M.; Park, Y. The role and effect of job satisfaction and empowerment on customers' perception of service quality: A study in the restaurant industry. J. Hosp. Tour. Res. 2010, 34, 56-77. [CrossRef]

45. Mathe, K.; Slevitch, L. An exploratory examination of supervisor undermining, employee involvement climate, and the effects on customer perceptions of service quality in quick-service restaurants. J. Hosp. Tour. Res. 2013, 37, 29-50. [CrossRef]

46. Kim, S.; Mattila, A.S. An examination of electronic video clips in the context of hotel Websites. Int. J. Hosp. Manag. 2011, 30, 612-618. [CrossRef] 